\title{
Enhanced Binding of an $N$-particle System Interacting with a Scalar Field II. Relativistic Version
}

\author{
by \\ Fumio Hiroshima and Itaru SASAKI
}

\begin{abstract}
An enhanced binding of $N$ relativistic particles coupled to a massless scalar Bose field is investigated. It is not assumed that the system has a ground state for the zero-coupling. It is shown, however, that there exists a ground state for sufficiently large coupling. The proof is based on checking the stability condition and showing uniform exponential decay of infrared-regularized ground states.
\end{abstract}

2010 Mathematics Subject Classification: Primary 81Q10; Secondary 62M15.

Keywords: Nelson model, ground state, enhanced binding.

\section{$\S 1$. Preliminaries}

\section{$\S 1.1$. Introduction}

Non-perturbative analysis of eigenvalues embedded in the continuous spectrum has been developed in the last decade and it has been applied to the mathematically rigorous analysis of the spectra of self-adjoint Hamiltonians in quantum field theory. Among other things, stability and instability of a quantum mechanical particle coupled to a quantum field have been investigated.

The Hamiltonian in quantum field theory is realized as a self-adjoint operator of the form

$$
K_{0}+\alpha K_{\mathrm{I}}
$$

Communicated by T. Kumagai. Received January 9, 2015. Revised May 23, 2015.

F. Hiroshima: Faculty of Mathematics, Kyushu University,

744 Motooka, Nishiku, Fukuoka, 819-0395 Japan;

e-mail: hiroshima@math.kyushu-u.ac.jp

I. Sasaki: Department of Mathematics, Shinshu University,

3-1-1- Asahi, Matsumoto, 390-8621 Japan;

e-mail: isasaki@shinshu-u.ac.jp

(C) 2015 Research Institute for Mathematical Sciences, Kyoto University. All rights reserved. 
acting in a Hilbert space over $\mathbb{C}$ for each value of the coupling constant $\alpha \in \mathbb{R}$. Here $K_{0}$ is the subject term and $K_{\mathrm{I}}$ an interaction term. We are concerned with ground states of $K_{0}+\alpha K_{\mathrm{I}}$ in this paper.

Let $\sigma(T)$ be the spectrum of a self-adjoint operator $T$.

Definition 1.1 (Ground state and ground state energy). Let $T$ be a self-adjoint operator bounded from below. Then the bottom of the spectrum, $E_{0}(T)=\inf \sigma(T)$, is called the ground state energy of $T$. Suppose $E_{0}(T)$ is an eigenvalue of $T$. Then an eigenvector $f$ associated with $E_{0}(T)$ is called a ground state of $T$, i.e., $T f=$ $E_{0}(T) f$.

Generally the bottom of the spectrum of the zero-coupling Hamiltonian $K_{0}$ is embedded in the continuous spectrum. Hence the spectral analysis of $K_{0}+\alpha K_{\mathrm{I}}$ is regarded as the perturbation problem of embedded eigenvalues. Although an analytic perturbation theory of the discrete spectrum is established for various types of self-adjoint operators, the perturbation of embedded eigenvalues is crucial and it is not straightforward to apply the perturbation theory of discrete spectra. Hence it is subtle to show the existence of a ground state of $K_{0}+\alpha K_{\mathrm{I}}$ even for small values of the coupling constant. Moreover a ground state need not exist for $K_{0}+\alpha K_{\mathrm{I}}, \alpha \neq 0$, even when $\inf \sigma\left(K_{0}+\alpha K_{\mathrm{I}}\right)>-\infty$ and $K_{0}$ has a ground state.

The existence or absence of a ground state for physically reasonable Hamiltonians of quantum field theory has been however proven so far under some assumptions. The existence of a ground state of the standard Nelson Hamiltonian [Nel64] was in particular proven in e.g. [BFS98, Spo98, Ger00, Sas05], where the most basic assumptions are

(1) the infrared regularity condition,

(2) the existence of a ground state of $K_{0}$.

In particular assumption (2) tells us that the Hamiltonians $K_{0}+\alpha K_{\mathrm{I}}$ also have a ground state for all $\alpha$.

It is found however that an interaction with quantum fields enhances the binding energy, which suggests that a Hamiltonian with sufficiently large coupling constant may have a ground state whether $K_{0}$ has a ground state or not. If $K_{0}+\alpha K_{\mathrm{I}}$ with sufficiently large coupling constant has a ground state whether $K_{0}$ has a ground state or not, then it is said that enhanced binding occurs. The study of enhanced binding was initiated by [HS01], and in [HS08] enhanced binding was shown for a system of $N$ nonrelativistic particles governed by a Schrödinger operator and linearly coupled to a massless scalar Bose field. In this paper we show enhanced binding after replacing the nonrelativistic particles with relativistic ones. 
Finally we make some comments on related work on enhanced binding. The enhanced binding has been studied so far for various kinds of models in quantum field theory. In [HS01] the enhanced binding of the Pauli-Fierz model with the dipole approximation is studied. In [HSS11] a complementary result to [HS01] is established, i.e., the absence of a ground state for a sufficiently small coupling constant. See also [AK03, BLV05, BV04, CEH03, CVV03, HVV03] for related work.

\section{§1.2. Main results}

The total Hamiltonian we consider is of the form

$$
H^{V}=H_{0}+\kappa H_{\mathrm{I}}
$$

The operator $H_{0}=H_{0}(\kappa)$ describes the zero-coupling Hamiltonian and is given by

$$
\begin{aligned}
& H_{0}=H_{\mathrm{p}}+\kappa^{2} H_{\mathrm{f}}, \\
& H_{\mathrm{p}}=\sum_{j=1}^{N}\left(\sqrt{-\Delta_{j}+m_{j}^{2}}-m_{j}+V\left(x_{j}\right)\right),
\end{aligned}
$$

where $m_{j}>0$ is the mass of the $j$-th particle, $V(x)$ an external potential, $H_{\mathrm{f}}$ the free field Hamiltonian, and $\kappa>0$ denotes a scaling parameter. The operator $H_{\mathrm{I}}$ describes a particle-boson linear interaction. We notice that there are no pair potentials in $H^{V}$, and $V$ is assumed to be independent of $j$ for simplicity. Introducing a dressing transformation $e^{i T}$ to derive an effective potential $V_{\text {eff }}$, we transform $H^{V}$ into

$$
e^{-i T} H^{V} e^{i T}=h_{\mathrm{eff}}^{V}+\kappa^{2} H_{\mathrm{f}}+H_{\mathrm{R}}(\kappa)
$$

where $h_{\text {eff }}^{V}$ is the effective particle Hamiltonian given by

$$
h_{\mathrm{eff}}^{V}=\sum_{j=1}^{N}\left(\sqrt{-\Delta_{j}+m_{j}^{2}}-m_{j}+V\left(x_{j}\right)\right)+V_{\mathrm{eff}}\left(x_{1}, \ldots, x_{N}\right)
$$

and $H_{\mathrm{R}}(\kappa)$ is a remainder term to be regarded as a perturbation of $h_{\mathrm{eff}}^{V}+\kappa^{2} H_{\mathrm{f}}$. Compensating for deriving $V_{\text {eff }}$ through the dressing transformation, we have the remainder term $H_{\mathrm{R}}(\kappa)$ which is unfortunately no longer linear and has the complicated form

$$
H_{\mathrm{R}}(\kappa)=\sum_{j=1}^{N}\left(\sqrt{\left(-i \nabla_{j}-\frac{1}{\kappa} A_{j}\left(x_{j}\right)\right)^{2}+m_{j}^{2}}-\sqrt{-\Delta_{j}+m_{j}^{2}}\right),
$$


where $A_{j}$ denotes some quantum vector field. Nevertheless it turns out to be a small perturbation for sufficiently large $\kappa$ in some sense.

We are interested in the existence of a ground state of $H^{V}$, equivalently of $e^{-i T} H^{V} e^{i T}$. We do not however assume the existence of ground states of $H_{0}$. As will be shown below, enhanced binding is exhibited by the transformed Hamiltonian (3) rather than $H^{V}$ itself. Since we consider a massless boson, the bottom of the spectrum of $H^{V}$ is the edge of the continuous spectrum and a regular perturbation cannot be applied. Thus it is not clear whether $e^{-i T} H^{V} e^{i T}$ has a ground state even when $h_{\text {eff }}^{V}$ has a ground state.

The conventional approach is to assume an infrared cutoff in the form of a factor $\hat{\lambda}$ in $H_{\mathrm{I}}$ by setting $\hat{\lambda}(k)\left\lceil_{|k|>\sigma}, H^{V}\right.$ with cutoff $\hat{\lambda}\left\lceil_{|k|>\sigma}\right.$ is denoted by $H_{\sigma}^{V}$, and to show the existence of a ground state $\Phi_{\sigma}$ of $H_{\sigma}^{V}$. The vector $\Phi_{\sigma}$ is called an infrared-regularized ground state. Then one is left to show that the sequence of ground states $\Phi_{\sigma}$ has a nonzero weak limit $\Phi$ as $\sigma \rightarrow 0$, which is the desired ground state of $H^{V}$. We show in this paper:

(A) the stability condition for $H^{V}$ is satisfied (Lemma 3.1),

(B) the infrared-regularized ground state $\Phi_{\sigma}$ has exponential decay uniformly with respect to the infrared cutoff parameter $\sigma$ (Lemma 3.8),

(C) (1) the stability condition and (2) exponential decay imply the existence of a ground state of $H^{V}$ (Appendix 4),

(D) there exist $\bar{\alpha}>0$ and $\kappa_{0}$ such that for each $\kappa>\kappa_{0}, H^{V}$ has a unique ground state for $|\alpha| \in(\bar{\alpha}, \bar{\alpha}(\kappa))$ with some $\bar{\alpha}(\kappa)$ (Theorem 2.3).

Statement (D) describes enhanced binding and is the main theorem in this paper.

\section{$\S 1.3$. Strategies}

We explain in more detail the technical improvements of this paper.

(Reduction to the stability condition for $h_{\mathrm{eff}}^{V}$ ) The stability condition is introduced in [GLL01] to show the existence of a ground state of nonrelativistic quantum electrodynamics. The key ingredient in this paper is that we show in

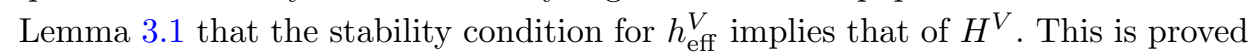
by applying an energy comparison inequality derived by functional integration of the heat semigroup generated by (3) (Lemma 3.2) and a simple variational principle (Lemma 3.3), hence we focus on showing the stability condition for $h_{\mathrm{eff}}^{V}$ instead of $H^{V}$.

(Uniform exponential localization by functional integration) Our method is a minor but nontrivial modification of [HS08] and a mixture of [Ger00, GLL01]. 
We do not assume the compactness of $H_{\mathrm{p}}$, unlike [Ger00]. Instead we show exponential localization of infrared-regularized ground states, $\left\|\Phi_{\sigma}(x)\right\|_{\mathscr{F}} \leq C_{\delta} e^{-\delta|x|}$, which is derived through functional integration in Lemma 3.8. The crucial point is to show that this localization is uniform in $\sigma>0$, i.e., $C_{\delta}$ and $\delta$ are independent of $\sigma>0$.

(Scaling) The scaling introduced in this paper can be obtained by replacing the annihilation operator $a$ and the creation operator $a^{*}$ with $\kappa a$ and $\kappa a^{*}$, respectively. This scaling is introduced in [Dav77, Dav79, Hir99] and the scaling limit as $\kappa \rightarrow \infty$ is called the weak coupling limit. Roughly speaking, at least in the nonrelativistic domain, $H_{\mathrm{p}} \cong-\frac{1}{2 m} \Delta+V$, and so

$$
H^{V}=\kappa^{2}\left(\kappa^{-2} H_{\mathrm{p}}+H_{\mathrm{f}}+\kappa^{-1} H_{\mathrm{I}}\right)
$$

with

$$
\kappa^{-2} H_{\mathrm{p}} \cong-\frac{1}{2 m \kappa^{2}} \Delta+\frac{1}{\kappa^{2}} V .
$$

Thus we interpret that enhanced binding of $H^{V}$ occurs when sufficiently large particle mass and shallow external potential are assumed. An alternative explanation of the scaling is that it is a tool to derive a Markov process from $e^{-t H^{V}}$. Although the scalar product $\left(f \otimes \Omega, e^{-t H^{V}} g \otimes \Omega\right)$ does not define a Markov process, $\left(f, e^{-t\left(h_{\text {eff }}-E_{\text {diag }}\right)} g\right)$ does with generator $h_{\text {eff }}-E_{\text {diag }}$. It can be obtained by the scaling limit:

$$
\left(f \otimes \Omega, e^{-t H^{V}} g \otimes \Omega\right) \rightarrow\left(f, e^{-t\left(h_{\text {eff }}-E_{\text {diag }}\right)} g\right)
$$

as $\kappa \rightarrow \infty$. This can be done in a similar manner to [Hir99]. More precisely, if $h_{\mathrm{eff}}$ has a unique strictly positive ground state $\phi_{\mathrm{p}}$, then there exists a diffusion process $\left(Y_{t}\right)_{t \geq 0}$ such that

$$
\left(f \phi_{\mathrm{p}}, e^{-t\left(h_{\mathrm{eff}}-E_{\mathrm{diag}}\right)} g \phi_{\mathrm{p}}\right)=\mathbb{E}\left[f\left(Y_{0}\right) g\left(Y_{t}\right)\right],
$$

where $\mathbb{E}$ denotes expectation, and $\left(Y_{t}\right)_{t \geq 0}$ is the so-called $P(\phi)_{1}$ process. See e.g. [GHPS12] for the construction of the $P(\phi)_{1}$ process.

The organization of this paper is as follows

In the remainder of Section 1 we define the Nelson model with a relativistic kinetic term, and introduce the scaling parameter $\kappa>0$. In Section 2 we introduce a dressing transformation, and mention the stability condition and uniform exponential decay of $\Phi_{\sigma}(x)$. In Section 3 we prove the stability condition in Section 3.1 and uniform exponential decay in Section 3.2, and in Section 3.3 we demonstrate enhanced binding.

In Appendix 4 we show that the relativistic version of the stability condition also implies the existence of a ground state. In Appendix 5 we review the fundamen- 
tal properties of the bottom of the essential spectrum of a relativistic Schrödinger operator. In Appendix 6 we give a functional integral representation of $e^{-t H^{V}}$ and prove an inequality used in the proof of exponential decay of infrared-regularized ground states. In Appendix 7 we derive an energy comparison inequality for the translation invariant Hamiltonian $\sum_{j=1}^{N}\left(\sqrt{-\Delta_{j}+m_{j}}-m_{j}+V\left(x_{j}\right)\right)$.

\section{§1.4. Definition}

We begin with the definition of the Nelson model with $N$ relativistic particles. Throughout we assume $N \geq 2$ and the dimension of the state space is $d \geq 3$. The Hamiltonian of the Nelson model can be realized as a self-adjoint operator on the tensor product of $L^{2}\left(\mathbb{R}^{d N}\right)$ and the boson Fock space $\mathscr{F}$ over $L^{2}\left(\mathbb{R}^{d}\right)$,

$$
\mathscr{H}=L^{2}\left(\mathbb{R}^{d N}\right) \otimes \mathscr{F} .
$$

Here $\mathscr{F}$ is defined by $\mathscr{F}=\bigoplus_{n=0}^{\infty} L_{\text {sym }}^{2}\left(\mathbb{R}^{d n}\right)$, where $L_{\text {sym }}^{2}\left(\mathbb{R}^{d n}\right)$ is the set of square integrable functions $\Psi$ on $\left(\mathbb{R}^{d}\right)^{n}$ such that $\Psi\left(x_{1}, \ldots, x_{n}\right)=\Psi\left(x_{\sigma(1)}, \ldots, x_{\sigma(n)}\right)$ for any permutation $\sigma$ of $\{1, \ldots, n\}$. A vector $\Psi \in \mathscr{F}$ is written as $\Psi=\left\{\Psi^{(n)}\right\}_{n=0}^{\infty}$ with $\Psi^{(n)} \in L_{\text {sym }}^{2}\left(\mathbb{R}^{d n}\right)$, and the Fock vacuum $\Omega \in \mathscr{F}$ is defined by $\Omega=\{1,0,0, \ldots\}$. We denote by $a(f)$ and $a^{*}(f), f \in L^{2}\left(\mathbb{R}^{d}\right)$, the annihilation and creation operators in $\mathscr{F}$, respectively. They satisfy the canonical commutation relations

$$
\left[a(f), a^{*}(g)\right]=(\bar{f}, g) \mathbb{1}, \quad[a(f), a(g)]=0=\left[a^{*}(f), a^{*}(g)\right]
$$

and the adjoint relation $a^{*}(f)=(a(\bar{f}))^{*}$. Throughout this paper, $(F, G)_{\mathcal{K}}$ denotes the scalar product on the Hilbert space $\mathcal{K}$, which is linear in $G$ and antilinear in $F$. We omit $\mathcal{K}$ unless confusion arises. We informally write $a^{\#}(f)=\int a^{\#}(k) f(k) d k$, $a^{\#}=a, a^{*}$. The second quantization of a closed operator $A$ on $L^{2}\left(\mathbb{R}^{d}\right)$ is denoted by $\mathrm{d} \Gamma(A)$. The free field Hamiltonian $H_{\mathrm{f}}$ is a self-adjoint operator on $\mathscr{F}$, given by the second quantization of the multiplication operator $\omega(k)=|k|$ on $L^{2}\left(\mathbb{R}^{d}\right)$ :

$$
H_{\mathrm{f}}=\mathrm{d} \Gamma(\omega) .
$$

Next we introduce the particle Hamiltonian. We suppose that $N$ relativistic particles are governed by the relativistic Schrödinger operator $H_{\mathrm{p}}$ of the form

$$
H_{\mathrm{p}}=\sum_{j=1}^{N}\left(\Omega_{j}+V_{j}\right)
$$

acting on $L^{2}\left(\mathbb{R}^{d N}\right)$, where

$$
\Omega_{j}=\Omega_{j}\left(p_{j}\right)=\sqrt{p_{j}^{2}+m_{j}^{2}}-m_{j}
$$


is the $j$-th particle Hamiltonian with momentum $p_{j}=-i \nabla_{x_{j}}$ and mass $m_{j}>0$. $V_{j}=V\left(x_{j}\right)$ denotes an external potential. In this paper, we assume for simplicity that there is no interparticle potential.

The Hamiltonian of the relativistic Nelson model is then defined by

$$
H^{V}=H_{0}+\kappa H_{\mathrm{I}},
$$

where the zero-coupling Hamiltonian $H_{0}$ is given by

$$
H_{0}=H_{\mathrm{p}} \otimes \mathbb{1}+\kappa^{2} \mathbb{1} \otimes H_{\mathrm{f}}
$$

and $\kappa>0$ is a scaling parameter. $H_{\mathrm{I}}$ denotes the linear interaction given by

$$
H_{\mathrm{I}}=\alpha \sum_{j=1}^{N} \int_{\mathbb{R}^{d N}}^{\oplus} \phi_{j}\left(x_{j}\right) d X
$$

under the identification $\mathscr{H} \cong \int_{\mathbb{R}^{d N}}^{\oplus} \mathscr{F} d X$, where $d X=d x_{1} \cdots d x_{N}$. Here $\alpha \geq 0$ is the coupling constant, and the scalar field $\phi_{j}(x)$ is given by

$$
\phi_{j}(x)=\overline{\frac{1}{\sqrt{2}} \int_{\mathbb{R}^{d}}\left(a^{*}(k) \hat{\lambda}_{j}(-k) e^{-i k x}+a(k) \hat{\lambda}_{j}(k) e^{i k x}\right) d k}
$$

for each $x \in \mathbb{R}^{d}$ with ultraviolet cutoff functions $\hat{\lambda}_{j}$. Here $\overline{\{\cdots\}}$ denotes operator closure. The standard choice of the ultraviolet cutoff is

$$
\hat{\lambda}_{j}(k)=(2 \pi)^{-d / 2} \omega(k)^{-1} \mathbb{1}_{|k| \leq \Lambda},
$$

where $\mathbb{1}_{X}$ denotes the characteristic function of $X$. We do not however fix any special cutoff function.

Throughout this paper we assume the following three conditions:

(V) $V(-\Delta+1)^{-1 / 2}$ is compact.

(UV) $\hat{\lambda}_{j}(-k)=\hat{\lambda}_{j}(k) \geq 0$ and $\hat{\lambda}_{j} \in L^{2}\left(\mathbb{R}^{d}\right)$ for $j=1, \ldots, N$.

(IR) $\hat{\lambda}_{j} / \omega \in L^{2}\left(\mathbb{R}^{d}\right)$ for $j=1, \ldots, N$.

Assumption (V) implies that $V$ is infinitesimally small with respect to the selfadjoint operator $\sqrt{-\Delta+m^{2}}-m$ for all $m \geq 0$. Hence, by the Kato-Rellich theorem, $H_{\mathrm{p}}$ is self-adjoint on $D\left(\sum_{j=1}^{N} \Omega_{j}\right)$ and essentially self-adjoint on any core for $\sum_{j=1}^{N} \Omega_{j}$, where $D(A)$ denotes the domain of $A$. (UV) implies that $H_{\mathrm{I}}$ is symmetric. Moreover (V), (UV) and (IR) also imply that, for all $\alpha \in \mathbb{R}$ and $\epsilon>0$,

$$
\left\|H_{\mathrm{I}} \Psi\right\| \leq \epsilon\left\|H_{0} \Psi\right\|+b_{\epsilon}\|\Psi\|, \quad \Psi \in D\left(H_{0}\right) .
$$

Therefore, by the Kato-Rellich theorem, $H^{V}$ is self-adjoint on $D\left(H_{0}\right)$ for all $\kappa>0$ and $\alpha \geq 0$. The nonnegativity $\hat{\lambda}_{j}(k) \geq 0$ in (UV) implies that the effective potential is attractive, which is used in Lemma 3.10. 


\section{§2. Existence of a ground state}

\section{§2.1. Dressing transformation}

To derive the effective particle Hamiltonian we introduce the so-called dressing transformation $e^{-i T}$, where $T=\frac{\alpha}{\kappa} \sum_{j=1}^{N} \pi_{j}$ and

$$
\left.\pi_{j}=\int_{\mathbb{R}^{d N}}^{\oplus} d X \overline{\left(\frac{i}{\sqrt{2}} \int\left(a^{*}(k) e^{-i k x_{j}} \frac{\hat{\lambda}_{j}(-k)}{\omega(k)}-a(k) e^{i k x_{j}} \frac{\hat{\lambda}_{j}(k)}{\omega(k)}\right) d k\right.}\right) .
$$

By (IR), $\pi_{j}$ is self-adjoint on $\mathscr{H}$ and so $e^{i T}$ is unitary.

Lemma 2.1. The unitary operator $e^{i T}$ maps $D\left(H^{V}\right)$ onto itself and

$$
e^{-i T} H^{V} e^{i T}=h_{\text {eff }}^{V} \otimes \mathbb{1}+\kappa^{2} \mathbb{1} \otimes H_{\mathrm{f}}+H_{\mathrm{R}}(\kappa),
$$

where the effective Hamiltonian is defined by

$$
h_{\mathrm{eff}}^{V}=\sum_{j=1}^{N}\left(\Omega_{j}+V_{j}\right)+V_{\mathrm{eff}},
$$

with the effective pair potential

$$
\begin{gathered}
V_{\mathrm{eff}}=\alpha^{2} \sum_{1 \leq i<j \leq N} W_{i j}\left(x_{i}-x_{j}\right), \\
W_{i j}(x)=-\int_{\mathbb{R}^{d}} \frac{\hat{\lambda}_{i}(-k) \hat{\lambda}_{j}(k)}{\omega(k)} e^{-i k x} d k .
\end{gathered}
$$

Here $H_{\mathrm{R}}(\kappa)$ is the remainder term given by

$$
\begin{aligned}
H_{\mathrm{R}}(\kappa) & =\sum_{j=1}^{N}\left(\Delta \Omega_{j}-\frac{\alpha^{2}}{2}\left\|\hat{\lambda}_{j} / \sqrt{\omega}\right\|^{2}\right), \\
\Delta \Omega_{j} & =\Omega_{j}\left(p_{j}+\frac{\alpha}{\kappa} A_{j}\right)-\Omega_{j}\left(p_{j}\right),
\end{aligned}
$$

with a vector field

$$
\begin{aligned}
A_{j} & =\left(A_{j 1}, \ldots, A_{j d}\right), \\
A_{j l} & =\int_{\mathbb{R}^{d N}}^{\oplus}\left(\frac{1}{\sqrt{2}} \int_{\mathbb{R}^{d}} k_{l}\left(a^{*}(k) e^{-i k x_{j}} \frac{\hat{\lambda}_{j}(-k)}{\omega(k)}+a(k) e^{i k x_{j}} \frac{\hat{\lambda}_{j}(k)}{\omega(k)}\right) d k\right) d X .
\end{aligned}
$$


Proof. We directly see that

$$
\begin{aligned}
e^{-i T} p_{j} e^{i T} & =p_{j}+\frac{\alpha}{\kappa} A_{j}, \\
e^{-i T} \phi_{j} e^{i T} & =\phi_{j}-\frac{\alpha}{\kappa} \sum_{i=1}^{N} \int_{\mathbb{R}^{d}} \frac{\hat{\lambda}_{i}(k) \hat{\lambda}_{j}(-k)}{\omega(k)} e^{-i k\left(x_{j}-x_{i}\right)} d k, \\
e^{-i T} H_{\mathrm{f}} e^{i T} & =H_{\mathrm{f}}-\frac{1}{\kappa} H_{\mathrm{I}}+\frac{\alpha^{2}}{2 \kappa^{2}} \sum_{i, j=1}^{N} \int_{\mathbb{R}^{d}} \frac{\hat{\lambda}_{i}(-k) \hat{\lambda}_{j}(k)}{\omega(k)} e^{-i k\left(x_{i}-x_{j}\right)} d k .
\end{aligned}
$$

From these, the lemma follows.

(UV) and (IR) imply that $V_{\text {eff }}$ is bounded. Therefore $H_{\text {eff }}^{V}$ is a self-adjoint operator on $D\left(\sum_{j=1}^{N} \Omega_{j}\right)$.

\section{$\S 2.2$. Main results}

Recall that $E_{0}(T)=\inf \sigma(T)$ for a self-adjoint operator $T$.

Theorem 2.2 (Existence of a ground state). Assume (V), (UV) and (IR) hold. Suppose that $E_{0}\left(h_{\mathrm{eff}}^{V}\right) \in \sigma_{\mathrm{disc}}\left(h_{\mathrm{eff}}^{V}\right)$. Then there exists $\kappa_{0}>0$ such that $H^{V}$ has a unique ground state for any $\kappa>\kappa_{0}$.

In order to show enhanced binding, we introduce an assumption on $V$ :

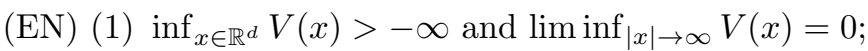

(2) $\sqrt{-\Delta}+N V$ acting in $L^{2}\left(\mathbb{R}^{d}\right)$ has a negative energy ground state;

(3) $V$ is a $d$-dimensional relativistic Kato-class potential, i.e.,

$$
\lim _{t \downarrow 0} \sup _{x \in \mathbb{R}^{d}} \mathbb{E}_{\mathrm{P}}^{x}\left[\int_{0}^{t} V\left(X_{s}\right) d s\right]=0
$$

where $\mathbb{E}_{\mathrm{P}}^{x}$ denotes the expectation on a probability space $\left(\mathcal{D}, \mathcal{B}, \mathrm{P}^{x}\right)$, and $\left(X_{t}\right)_{t>0}$ denotes the $d$-dimensional Lévy process with characteristic function $\mathbb{E}_{\mathrm{P}}^{x}\left[e^{i u X_{t}}\right]=e^{-t\left(\sqrt{u^{2}+m^{2}}-m\right)} e^{i u x}$.

Assumption (EN)(1) is used only to show spatial exponential decay of the infraredregularized ground state $\Phi_{\sigma}$. The second assumption $(\mathrm{EN})(2)$, which is used in (55), is crucial to showing enhanced binding. Intuitively a sufficiently strong interaction engages $N$ particles through linear interaction of the quantum field, and consequently the total Hamiltonian can be regarded as $\sqrt{-\Delta}+N V$. This intuitive description is justified in this paper. $(\mathrm{EN})(3)$ is used to show the continuity of ground state energy of a translation invariant Hamiltonian in Lemma 3.11.

We now state the main results of this paper. 
Theorem 2.3 (Enhanced binding). Suppose (V), (UV) and (IR) hold. Assume (EN) and $N \geq 2$. Then there exist $\bar{\alpha}, \kappa_{0}>0$ such that for each $\kappa>\kappa_{0}, H^{V}$ has a unique ground state for $|\alpha| \in(\bar{\alpha}, \bar{\alpha}(\kappa))$ with some constant $\bar{\alpha}(\kappa)$.

Remark 2.4. In Theorem 2.2, $h_{\text {eff }}^{V}$ has a ground state. In Theorem 2.3 we do not assume the existence of a ground state of $H_{\mathrm{p}}$, i.e., the zero-coupling Hamiltonian $H_{0}$ does not necessarily have a ground state.

Remark 2.5. In the case of $N=1$, we cannot apply our method to show enhanced binding. Although in this case enhanced binding may also occur, it is crucial to estimate the dressing transformed Hamiltonian (14). We do not discuss this case later.

Example 2.6. We give examples of $V$ satisfying (V) and (EN), but for which $\sqrt{-\Delta+1}-1+V$ has no ground state in dimension $d \geq 3$. Suppose that

$$
|\tilde{V}(x)| \leq c(1+|x|)^{-\epsilon}
$$

with some $c, \epsilon>0$. For example $\tilde{V}=-e^{-x^{2}}$. Then $(\mathrm{V})$ is satisfied with $V=\delta \tilde{V}$ for all constants $\delta>0$. Let $\tilde{V} \not \equiv 0, \tilde{V} \leq 0$ and $\tilde{V} \in L^{d}\left(\mathbb{R}^{d}\right) \cap L^{d / 2}\left(\mathbb{R}^{d}\right)$. Let $\delta>0$ be a sufficiently small constant and set

$$
H_{\delta}=\sqrt{-\Delta+1}-1+\delta \tilde{V} .
$$

Let $E_{\delta}(\cdot)$ be the spectral measure of $H_{\delta}$. Since $\tilde{V}(\sqrt{-\Delta+1})^{-1}$ is compact, the essential spectrum of $H_{\delta}$ is $\sigma_{\text {ess }}\left(H_{\delta}\right)=[0, \infty)$ for all $\delta>0$. By the relativistic version of the Lieb-Thirring bound [Dau83], we have

$$
\operatorname{dim} \operatorname{Ran} E_{\delta}((-\infty, 0]) \leq c_{1} \delta^{d} \int_{\mathbb{R}^{d}}|\tilde{V}(x)|^{d} d x+c_{2} \delta^{d / 2} \int_{\mathbb{R}^{d}}|\tilde{V}(x)|^{d / 2} d x,
$$

where $c_{1}$ and $c_{2}$ are positive constants independent of $\tilde{V}$. Hence $H_{\delta}$ has no ground state for sufficiently small $\delta$ such that the right-hand side of (21) is strictly smaller than one. Similarly $\sigma_{\text {ess }}(\sqrt{-\Delta}+N \delta \tilde{V})=[0, \infty)$. However, $\sqrt{-\Delta}+N \delta \tilde{V}$ has a negative eigenvalue for sufficiently large $N$, since $\inf \sigma(\sqrt{-\Delta}+N \delta \tilde{V})<0$ for sufficiently large $N$, which implies that $\sqrt{-\Delta}+N \delta \tilde{V}$ has a ground state for sufficiently large $N$. Therefore for sufficiently small $\delta, V=\delta \tilde{V}$ satisfies (V) and (EN), but $\sqrt{-\Delta+1}-1+\delta \tilde{V}$ has no ground state.

\section{$\S 2.3$. Stability condition and exponential decay}

In order to prove Theorems 2.2 and 2.3 we investigate the stability condition. First of all we introduce cluster Hamiltonians. Let $C_{N}=\{1, \ldots, N\}$. For each $\beta \subset C_{N}$ 
$(\beta \neq \emptyset)$, we define

$$
\begin{aligned}
H^{0}(\beta) & =\sum_{j \in \beta}\left(\Omega_{j}+\kappa \alpha \phi_{j}\right)+\kappa^{2} H_{\mathrm{f}}, \\
H^{V}(\beta) & =H^{0}(\beta)+\sum_{j \in \beta} V_{j},
\end{aligned}
$$

acting on $L^{2}\left(\mathbb{R}^{d|\beta|}\right) \otimes \mathscr{F}$, where $\phi_{j}=\int_{\mathbb{R}^{d|\beta|} \mid}^{\oplus} \phi_{j}\left(x_{j}\right) d X_{\beta}, X_{\beta}=\left(x_{j}\right)_{j \in \beta}$. Clearly $H^{V}=H^{V}\left(C_{N}\right)$. Let

$$
E^{0}(\beta)=\inf \sigma\left(H^{0}(\beta)\right), \quad E^{V}(\beta)=\inf \sigma\left(H^{V}(\beta)\right) .
$$

For $\beta=\emptyset$, we set $E^{0}(\emptyset)=E^{V}(\emptyset)=0$. The lowest two-cluster threshold is defined as the minimal energy of systems such that only the particles involved in $\beta$ are bound by the origin but others are sufficiently far from the origin. It is defined by

$$
\Sigma^{V}=\min \left\{E^{V}(\beta)+E^{0}\left(\beta^{c}\right) \mid \beta \subsetneq C_{N}\right\} .
$$

The gap between the ground state energy $E^{V}$ and the lowest two-cluster threshold $\Sigma^{V}$ is related to the existence of a ground state by the proposition below. Let $H_{\sigma}^{V}$ be $H^{V}$ with $\hat{\lambda}_{j}$ replaced by $\hat{\lambda}_{j}(k) \mathbb{1}_{|k|>\sigma}$.

Proposition 2.7. (Case $\sigma>0$ ) Suppose that $E^{V}<\Sigma^{V}$. Then $H_{\sigma}^{V}$ has a unique ground state $\Phi_{\sigma}$.

(Case $\sigma=0)$ Suppose that $E^{V}<\Sigma^{V}$ and there exists $\delta>0$ independent of $\sigma$ such that $\sup _{0<\sigma<\bar{\sigma}}\left\|\left(e^{\delta|X|} \otimes \mathbb{1}\right) \Phi_{\sigma}\right\|_{\mathscr{H}}<\infty$ with some $\bar{\sigma}>0$. Then $H^{V}$ has a ground state.

Proof. The proof is a minor modification of those in [Ger00, GLL01], and it is given in Appendix 4.1 for $\sigma>0$, and in Appendix 4.2 for the case $\sigma=0$.

The condition $\Sigma^{V}>E^{V}$ is called the stability condition. For our model the uniform exponential decay of $\left\|\Phi_{\sigma}(x)\right\|_{\mathscr{F}}$ may be derived from the stability condition, but we do not prove it. So we need not only the stability condition but also uniform exponential decay.

\section{$\S 3$. Proof of the main theorem}

In order to show Theorems 2.2 and 2.3, by Proposition 2.7 it is enough to show both (1) the stability condition and (2) the uniform exponential decay of $\left\|\Phi_{\sigma}(x)\right\|_{\mathscr{F}}$.

\section{$\S 3.1$. Stability condition}

It is not straightforward to show the stability condition, so we will make a detour and the discussion will be reduced to that of the effective particle Hamiltonian $h_{\text {eff }}^{V}$. 
Let us define the lowest two-cluster threshold of $h_{\text {eff }}^{V}$ in a similar way to $H^{V}$ and we shall compare it with $\Sigma^{V}$. For $\beta \subset C_{N}$, we define effective cluster Hamiltonians by

$$
\begin{aligned}
& h_{\mathrm{eff}}^{0}(\beta)=\sum_{j \in \beta} \Omega_{j}-\alpha^{2} \sum_{i, j \in \beta, i<j} W_{i j}\left(x_{i}-x_{j}\right), \\
& h_{\mathrm{eff}}^{V}(\beta)=h_{\mathrm{eff}}^{0}(\beta)+\sum_{j \in \beta} V_{j} .
\end{aligned}
$$

We set

$$
\mathcal{E}^{0}(\beta)=\inf \sigma\left(h_{\text {eff }}^{0}(\beta)\right), \quad \mathcal{E}^{V}(\beta)=\inf \sigma\left(h_{\text {eff }}^{V}(\beta)\right)
$$

and $\mathcal{E}^{V}=\mathcal{E}^{V}\left(C_{N}\right)$. Then the lowest two-cluster threshold of $h_{\text {eff }}^{V}$ is defined by

$$
\Xi^{V}=\min \left\{\mathcal{E}^{V}(\beta)+\mathcal{E}^{0}\left(\beta^{c}\right) \mid \beta \subsetneq C_{N}\right\} .
$$

Constants $c^{V}$ and $d^{V}$ are such that $\left\|\sum_{j=1}^{N} \Omega_{j} \Psi\right\| \leq c^{V}\left\|h_{\text {eff }}^{V} \Psi\right\|+d^{V}\|\Psi\|$ and we set

$$
\begin{aligned}
\mathcal{G}(t)= & \left(\sum_{j=1}^{N}\left\|\hat{\lambda}_{j} / \omega\right\|\left\|\hat{\lambda}_{j}\right\|\right) t^{2}+\left(\sum_{j=1}^{N} \sqrt{2} m_{j}\left\|\hat{\lambda}_{j} / \omega\right\|\right)|t| \\
& +\sqrt{2} N\left(c^{V}\left|\mathcal{E}^{V}\right|+d^{V}\right) .
\end{aligned}
$$

The next lemma is a key ingredient of this paper.

Lemma 3.1. Assume that $\Xi^{V}-\mathcal{E}^{V}>0$, and $\alpha$ and $\kappa$ satisfy $\Xi^{V}-\mathcal{E}^{V}>\mathcal{G}(\alpha / \kappa)$. Then the stability condition $\Sigma^{V}-E^{V}>0$ holds.

In order to prove Lemma 3.1, we prepare two lemmas. We set

$$
E_{\text {diag }}=\frac{\alpha^{2}}{2} \sum_{j=1}^{N}\left\|\hat{\lambda}_{j} / \sqrt{\omega}\right\|^{2}
$$

Lemma 3.2. For all $\beta \subset C_{N}$,

$$
E^{\#}(\beta) \leq \mathcal{E}^{\#}(\beta)+\frac{\alpha^{2}}{2} \sum_{j \in \beta}\left\|\hat{\lambda}_{j} / \sqrt{\omega}\right\|^{2}, \quad \#=0, V .
$$

In particular, $\Xi^{V} \leq \Sigma^{V}+E_{\text {diag }}$.

Proof. See Proposition 6.3.

Lemma 3.3. For all $\kappa>0$ we have $E^{V} \leq \mathcal{E}^{V}+\mathcal{G}(\alpha / \kappa)-E_{\text {diag }}$. 
Proof. For every $\epsilon>0$, we can choose a normalized vector $v \in C_{0}^{\infty}\left(\mathbb{R}^{d N}\right)$ such that $\left\|\left(h_{\text {eff }}^{V}-\mathcal{E}^{V}\right) v\right\| \leq \epsilon$. Set $\Psi=v \otimes \Omega$. Then, by Lemma 2.1, we have

$$
E^{V} \leq \mathcal{E}^{V}+\epsilon+\left(\Psi,\left(-E_{\text {diag }}+\sum_{j=1}^{N} \Delta \Omega_{j}\right) \Psi\right)
$$

Since $\pi_{j}$ commutes with $p_{i}, i \neq j$, by setting $T_{j}=\alpha \pi_{j} / \kappa$ we can see that $\Delta \Omega_{j}=$ $e^{-i T_{j}} \Omega_{j} e^{i T_{j}}-\Omega_{j}$ and

$$
\left|\left(\Psi, \Delta \Omega_{j} \Psi\right)\right|=\left|\left(\left(e^{i T_{j}}-1\right) \Psi, \Omega_{j} e^{i T_{j}} \Psi\right)+\left(\Psi, \Omega_{j}\left(e^{i T_{j}}-1\right) \Psi\right)\right| .
$$

Hence

$$
\left|\left(\Psi, \Delta \Omega_{j} \Psi\right)\right| \leq \frac{|\alpha|}{\kappa}\left\|\pi_{j} \Psi\right\| \cdot\left\|\Omega_{j} e^{i T_{j}} \Psi\right\|+\frac{|\alpha|}{\kappa}\left\|\pi_{j} \Psi\right\| \cdot\left\|\Omega_{j} \Psi\right\| .
$$

The right-hand side above is identical with

$$
\frac{|\alpha|}{\sqrt{2} \kappa}\left\|\hat{\lambda}_{j} / \omega\right\|\left(\left(\Psi,\left(p_{j}+\frac{|\alpha|}{\kappa} A_{j}\right)^{2} \Psi\right)^{1 / 2}+\left(\Psi, p_{j}^{2} \Psi\right)^{1 / 2}\right) .
$$

Thus

$$
\left|\left(\Psi, \Delta \Omega_{j} \Psi\right)\right| \leq \frac{|\alpha|}{\sqrt{2} \kappa}\left\|\hat{\lambda}_{j} / \omega\right\|\left(2\left\|\Omega_{j} \Psi\right\|+2 m_{j}+\frac{\sqrt{2}|\alpha|}{\kappa}\left\||k| \hat{\lambda}_{j} / \omega\right\|\right)
$$

and

$$
\begin{aligned}
E^{V} \leq & \mathcal{E}^{V}+\epsilon+\sum_{j=1}^{N} \frac{|\alpha|}{\sqrt{2} \kappa}\left\|\hat{\lambda}_{j} / \omega\right\|\left(2 m_{j}+\frac{\sqrt{2}|\alpha|}{\kappa}\left\||k| \hat{\lambda}_{j} / \omega\right\|\right) \\
& +\sum_{j=1}^{N} \frac{\sqrt{2}|\alpha|}{\kappa}\left\|\hat{\lambda}_{j} / \omega\right\|\left(c^{V}\left(\left|\mathcal{E}^{V}\right|+\epsilon\right)+d^{V}\right)-E_{\text {diag }} .
\end{aligned}
$$

Since $\epsilon>0$ is arbitrary, the lemma follows.

Proof of Lemma 3.1. By Lemmas 3.2 and 3.3, we have

$$
\Sigma^{V}-E^{V} \geq \Xi^{V}-\mathcal{E}^{V}-\mathcal{G}(\alpha / \kappa)>0 .
$$

\section{§3.2. Exponential decay}

Functional integration has proven to be a strong tool to show exponential localization of a bound state in quantum mechanics. That can also be applied in quantum field theory.

Let $\left(X_{t}\right)_{t \geq 0}=\left(X_{t}^{1}, \ldots, X_{t}^{N}\right)_{t \geq 0}$ be $N$ independent $d$-dimensional Lévy processes on a probability space $\left(\mathcal{D}, \mathcal{B}, \mathrm{P}^{x}\right), x \in \mathbb{R}^{d N}$, with characteristic function

$$
\mathbb{E}_{\mathrm{P}}^{0}\left[e^{-i u \cdot X_{t}}\right]=e^{-t \sum_{j=1}^{N}\left(\sqrt{u_{j}^{2}+m_{j}^{2}}-m_{j}\right)}, \quad u=\left(u_{1}, \ldots, u_{N}\right) \in \mathbb{R}^{d N} .
$$


Here and in what follows, $\mathbb{E}_{m}^{x}[\cdots]$ denotes expectation with respect to a path measure $m^{x}$ starting from $x$. Let $W_{\text {eff }}=W_{\text {eff }}\left(x_{1}, \ldots, x_{N}\right)=\sum_{j=1}^{N} V\left(x_{j}\right)+V_{\text {eff }}(x)$.

Proposition 3.4. There exists $\sigma_{0}>0$ such that for all $\sigma \leq \sigma_{0}$,

$$
\left\|\Phi_{\sigma}(X)\right\|_{\mathscr{F}} \leq e^{t\left(E^{V}+E_{\mathrm{diag}}+\epsilon(\sigma)\right)}\left(\mathbb{E}_{\mathrm{P}}^{X}\left[e^{-2 \int_{0}^{t} W_{\mathrm{eff}}\left(X_{s}\right) d s}\right]\right)^{1 / 2}\left\|\Phi_{\sigma}\right\|_{\mathscr{H}}
$$

for each $X \in \mathbb{R}^{d N}$, where $\epsilon(\sigma)>0$ satisfies $\lim _{\sigma \rightarrow 0} \epsilon(\sigma)=0$.

Proof. See Proposition 6.4.

From Proposition 3.4, in order to show the exponential decay of $\left\|\Phi_{\sigma}(X)\right\|_{\mathscr{F}}$ it suffices to estimate

$$
e^{t\left(E^{V}+E_{\text {diag }}\right)} \mathbb{E}_{\mathrm{P}}^{X}\left[e^{-2 \int_{0}^{t} W_{\text {eff }}\left(X_{s}\right) d s}\right]^{1 / 2}
$$

To do so, we divide $W_{\text {eff }}$ into two parts. Let

$$
B_{R}=\left\{x=\left(x_{1}, \ldots, x_{N}\right) \in \mathbb{R}^{d N}|| x \mid \geq 2 R \text { and } \min \left\{\left|x_{i}-x_{j}\right|, i \neq j\right\} \leq|x| / 2\right\} .
$$

Define $V_{\mathrm{eff}, \infty}^{R}=V_{\mathrm{eff}} \mathbb{1}_{B_{R}}$ and $V_{\mathrm{eff}, 0}^{R}=V_{\mathrm{eff}} \mathbb{1}_{B_{R}^{c}}$. Then

$$
W_{\text {eff }}=V+V_{\text {eff }, 0}^{R}+V_{\text {eff }, \infty}^{R} .
$$

By the Riemann-Lebesgue lemma, $\lim _{|x| \rightarrow \infty} W_{i j}(x)=0$. Notice that

$$
\begin{aligned}
& \lim _{|x| \rightarrow \infty}\left(V(x)+V_{\mathrm{eff}, 0}^{R}(x)\right)=0, \\
& \left\|V_{\mathrm{eff}, \infty}^{R}\right\|_{\infty} \leq \frac{\alpha^{2}}{2} \sum_{i \neq j} \int \frac{\hat{\lambda}_{i}(k) \hat{\lambda}_{j}(-k)}{\omega(k)} d k .
\end{aligned}
$$

The Lévy measure $\nu_{j}(d x)=\nu_{j}(x) d x$ associated with the Lévy process $\left(X_{t}^{j}\right)_{t \geq 0}$ is given by

$$
\nu_{j}(x)=2\left(\frac{m_{j}}{2 \pi}\right)^{(d+1) / 2} \frac{1}{|x|^{(d+1) / 2}} \int_{0}^{\infty} \xi^{(d-1) / 2} e^{-\frac{1}{2}\left(\xi+\xi^{-1}\right) m_{j}|x|} d \xi, \quad x \in \mathbb{R}^{d} .
$$

We note that $\nu_{j}(x) \leq C e^{-c|x|}$ with some constants $C, c \geq 0$.

Proposition 3.5. There exist $\eta, C_{1}, C_{2}>0$ such that

$$
\mathrm{P}^{0}\left(\sup _{0 \leq s \leq t}\left|X_{s}\right|>a\right) \leq C_{1} e^{-\eta a} e^{C_{2} t} \quad \text { for all } a>0 .
$$

Proof. We see that

$$
\mathrm{P}^{0}\left(\sup _{0 \leq s \leq t}\left|X_{s}\right|>a\right)=\mathbb{E}_{\mathrm{P}}^{0}\left[\mathbb{1}_{\sup _{0 \leq s \leq t}\left|X_{s}\right|-a>0}\right] \leq e^{-\eta a} \mathbb{E}_{\mathrm{P}}^{0}\left[e^{\eta \sup _{0 \leq s \leq t}\left|X_{s}\right|}\right] .
$$


It is known that $\mathbb{E}_{\mathrm{P}}^{0}\left[e^{\eta \sup _{0 \leq s \leq t}\left|X_{s}\right|}\right]<C_{1} e^{C_{2} t}$ for sufficiently small $\eta>0$ [CMS90]. Hence the proposition follows.

We define $\mathscr{B}=\left\{X_{s} \in B_{R}^{c}\right.$ for all $\left.0 \leq s \leq t\right\}$. Since $V_{\text {eff }, \infty}^{R}\left(X_{s}\right)=0$ on $\mathscr{B}$, we have

$$
\begin{aligned}
& \mathbb{E}_{\mathrm{P}}^{X}\left[e^{-2 \int_{0}^{t} W_{\text {eff }}\left(X_{s}\right) d s}\right] \\
& \quad=\mathbb{E}_{\mathrm{P}}^{X}\left[\mathbb{1}_{\mathscr{B}} e^{-2 \int_{0}^{t}\left(V+V_{\text {eff }, 0}^{R}\right)\left(X_{s}\right) d s}\right]+\mathbb{E}_{\mathrm{P}}^{X}\left[\mathbb{1}_{\mathscr{B}} e^{-2 \int_{0}^{t} W_{\text {eff }}\left(X_{s}\right) d s}\right] .
\end{aligned}
$$

By the Schwarz inequality,

$$
\begin{aligned}
\mathbb{E}_{\mathrm{P}}^{X} & {\left[\mathbb{1}_{\mathscr{B}^{c}} e^{-2 \int_{0}^{t} W_{\text {eff }}\left(X_{s}\right) d s}\right] } \\
& \leq \mathbb{E}_{\mathrm{P}}^{X}\left[\mathbb{1}_{\mathscr{B}^{c}} e^{-4 \int_{0}^{t} V_{\text {eff }, \infty}^{R}\left(X_{s}\right) d s}\right]^{1 / 2} \mathbb{E}_{\mathrm{P}}^{X}\left[\mathbb{1}_{\mathscr{B}^{c}} e^{-4 \int_{0}^{t}\left(V+V_{\text {eff }, 0}^{R}\right)\left(X_{s}\right) d s}\right]^{1 / 2}
\end{aligned}
$$

We will estimate the terms in (39) and (40). Set

$$
\begin{aligned}
W_{a}^{R}(x) & =\inf \left\{V(y)+V_{\mathrm{eff}, \infty}^{R}(y)|| x-y \mid<a\right\}, \\
W_{\infty}^{R} & =\inf _{x \in \mathbb{R}^{d N}}\left(V(x)+V_{\mathrm{eff}, \infty}^{R}(x)\right) .
\end{aligned}
$$

Lemma 3.6. Suppose $(\mathrm{EN})(1)$ holds. Let $R, a>0$. Then for all $X \in \mathbb{R}^{d N}$ and $t>0$,

$$
\mathbb{E}_{\mathrm{P}}^{X}\left[e^{-2 \int_{0}^{t}\left(V\left(X_{s}\right)+V_{\mathrm{eff}, \infty}^{R}\left(X_{s}\right)\right) d s}\right] \leq e^{-2 t W_{a}^{R}(x)}+C_{1} e^{-2 t W_{\infty}^{R}} e^{C_{2} t} e^{-\eta a},
$$

where $C_{1}, C_{2}$ and $\eta$ are as in (38).

Proof. Set $A=\left\{\sup _{0<s<t}\left|X_{s}\right|<a\right\} \subset \mathcal{D}$. Since $\left(X_{t}\right)_{t>0}$ under the probability measure $\mathrm{P}^{X}$ and $\left(X_{t}+X\right)_{t \geq 0}$ under $\mathrm{P}^{0}$ are identically distributed, we have

$$
\mathbb{E}_{\mathrm{P}}^{X}\left[e^{-2 \int_{0}^{t}\left(V\left(X_{s}\right)+V_{\mathrm{eff}, \infty}^{R}\left(X_{s}\right)\right) d s}\right]=\mathbb{E}_{\mathrm{P}}^{0}\left[e^{-2 \int_{0}^{t}\left(V\left(X_{s}+X\right)+V_{\text {eff }, \infty}^{R}\left(X_{s}+X\right)\right) d s}\right] .
$$

Then

$$
\begin{aligned}
& \mathbb{E}_{\mathrm{P}}^{0}\left[\mathbb{1}_{A} e^{-2 \int_{0}^{t}\left(V\left(X_{s}+X\right)+V_{\mathrm{eff}, \infty}^{R}\left(X_{s}+X\right)\right) d s}\right] \leq e^{-2 t W_{a}^{R}(x)}, \\
& \mathbb{E}_{\mathrm{P}}^{0}\left[\mathbb{1}_{A^{c}} e^{-2 \int_{0}^{t}\left(V\left(X_{s}+X\right)+V_{\mathrm{eff}, \infty}^{R}\left(X_{s}+X\right)\right) d s}\right] \leq e^{-2 t W_{\infty}^{R}} \mathbb{E}_{\mathrm{P}}^{0}\left[\mathbb{1}_{A^{c}}\right] \leq e^{-2 t W_{\infty}^{R} C_{1} e^{C_{2} t} e^{-\eta a}}
\end{aligned}
$$

by Proposition 3.5, and the lemma follows.

Lemma 3.7. Let $X \in \mathbb{R}^{d N}$ and set $R=|X|$. Then

$$
\mathbb{E}_{\mathrm{P}}^{X}\left[\mathbb{1}_{\mathscr{B}} e^{-4 \int_{0}^{t} V_{\mathrm{eff}, \infty}^{R}\left(X_{s}\right) d s}\right] \leq e^{4\left\|V_{\mathrm{eff}, \infty}\right\|_{\infty} t} C_{1} e^{C_{2} t} e^{-\eta R}
$$

where $C_{1}, C_{2}$ and $\eta$ are as in (38).

Proof. Since $\mathbb{E}_{\mathrm{P}}^{X}\left[e^{-4 \int_{0}^{t} V_{\mathrm{eff}, \infty}^{R}\left(X_{s}\right) d s}\right] \leq \mathbb{E}_{\mathrm{P}}^{X}\left[e^{4\left\|V_{\mathrm{eff}, \infty}\right\|_{\infty} \int_{0}^{t} \mathbb{1}_{B_{R}}\left(X_{s}\right) d s}\right]$, we can see that 


$$
\begin{aligned}
\mathbb{E}_{\mathrm{P}}^{X} & {\left[e^{\left.-4 \int_{0}^{t} V_{\mathrm{eff}, \infty}^{R}\left(X_{s}\right) d s\right]}\right.} \\
& \leq \sum_{n=0}^{\infty} \frac{\left(4\left\|V_{\mathrm{eff}, \infty}\right\|_{\infty}\right)^{n}}{n !} \int_{0}^{t} d s_{1} \cdots \int_{0}^{t} d s_{n} \mathbb{E}_{\mathrm{P}}^{X}\left[\mathbb{1}_{\mathscr{B}^{c}} \prod_{j=1}^{n} \mathbb{1}_{B_{R}}\left(X_{s_{j}}\right)\right] \\
& =\mathbb{E}_{\mathrm{P}}^{X}\left[\mathbb{1}_{\mathscr{B}^{c}}\right]+\sum_{n=1}^{\infty} \frac{\left(4\left\|V_{\mathrm{eff}, \infty}\right\|_{\infty}\right)^{n}}{n !} \int_{0}^{t} d s_{1} \cdots \int_{0}^{t} d s_{n} \mathbb{E}_{\mathrm{P}}^{0}\left[\mathbb{1}_{\mathscr{B}^{c}} \prod_{j=1}^{n} \mathbb{1}_{B_{R}}\left(X+X_{s_{j}}\right)\right] .
\end{aligned}
$$

Now,

$$
\begin{aligned}
\mathbb{E}_{\mathrm{P}}^{X}\left[\mathbb{1}_{\mathscr{B}^{c}}\right] & \leq \mathrm{P}^{0}\left(\sup _{0 \leq s \leq t}\left|X_{s}+X\right|>2 R\right) \\
& \leq \mathrm{P}^{0}\left(\sup _{0 \leq s \leq t}\left|X_{s}\right|>2 R-|X|\right)=\mathrm{P}^{0}\left(\sup _{0 \leq s \leq t}\left|X_{s}\right|>R\right)
\end{aligned}
$$

By the definition of $B_{R}$, in a similar way we have

$$
\begin{aligned}
\mathbb{E}_{\mathrm{P}}^{X}\left[e^{-4 \int_{0}^{t} V_{\mathrm{eff}, \infty}^{R}\left(X_{s}\right) d s}\right] \leq \mathrm{P}^{X}\left(\mathscr{B}^{c}\right)+\sum_{n=1}^{\infty} \frac{\left(4\left\|V_{\mathrm{eff}, \infty}\right\|_{\infty}\right)^{n}}{n !} \\
\quad \times \int_{0}^{t} d s_{1} \ldots \int_{0}^{t} d s_{n} \mathrm{P}^{0}\left(\left|X_{s_{1}}+X\right|>2 R, \ldots,\left|X_{s_{n}}+X\right|>2 R\right) \\
\leq \mathrm{P}^{X}\left(\mathscr{B}^{c}\right)+\sum_{n=1}^{\infty} \frac{\left(4\left\|V_{\mathrm{eff}, \infty}\right\|_{\infty}\right)^{n}}{n !} \int_{0}^{t} d s_{1} \cdots \int_{0}^{t} d s_{n} \mathrm{P}^{0}\left(\left|X_{s_{1}}\right|>R, \ldots,\left|X_{s_{n}}\right|>R\right) .
\end{aligned}
$$

From $\mathrm{P}^{0}\left(\left|X_{s_{1}}\right|>R, \ldots,\left|X_{s_{n}}\right|>R\right) \leq \mathrm{P}^{0}\left(\sup _{0 \leq s \leq t}\left|X_{s}\right|>R\right)$ and Proposition 3.5, we have

$$
\begin{aligned}
& \mathbb{E}_{\mathrm{P}}^{X}\left[e^{-4 \int_{0}^{t} V_{\text {eff }, \infty}^{R}\left(X_{s}\right) d s}\right] \\
& \leq \mathrm{P}^{0}\left(\sup _{0 \leq s \leq t}\left|X_{s}\right|>R\right)+\sum_{n=1}^{\infty} \frac{\left(4\left\|\mid V_{\mathrm{eff}, \infty}\right\|_{\infty}\right)^{n}}{n !} \int_{0}^{t} d s_{1} \ldots \int_{0}^{t} d s_{n} \mathrm{P}^{0}\left(\sup _{0 \leq s \leq t}\left|X_{s}\right|>R\right) \\
& \leq \sum_{n=0}^{\infty} \frac{\left(4\left\|V_{\mathrm{eff}, \infty}\right\|_{\infty}\right)^{n}}{n !} t^{n} C_{1} e^{C_{2} t} e^{-\eta R}=e^{4\left\|V_{\mathrm{eff}, \infty}\right\|_{\infty} t} C_{1} e^{C_{2} t} e^{-\eta R} .
\end{aligned}
$$

Hence the lemma follows.

Lemma 3.8. Let $\Phi_{\sigma}$ be the infrared-regularized ground state. Suppose (EN)(1) holds and $E^{V}+E_{\text {diag }}<0$. Furthermore assume that $E^{V}+E_{\text {diag }}+\epsilon(\sigma)<-\gamma$ with some $\gamma>0$ for $\sigma<\bar{\sigma}$, where $\epsilon(\sigma)$ is as in Proposition 3.4. Then there exist $\delta, C_{\delta}>0$ independent of $\sigma$ such that

$$
\sup _{0<\sigma<\bar{\sigma}}\left\|\Phi_{\sigma}(X)\right\|_{\mathscr{F}} \leq C_{\delta} e^{-\delta \min \{\gamma, \eta\}|X|},
$$

where $\eta>0$ is as in Proposition 3.5 . 
Proof. We set $\tilde{E}=E^{V}+E_{\text {diag }}+\epsilon(\sigma)$. It is enough to estimate

$$
e^{2 t \tilde{E}} \mathbb{E}_{\mathrm{P}}^{X}\left[e^{-2 \int_{0}^{t} W_{\mathrm{eff}}\left(X_{s}\right) d s}\right]
$$

by Proposition 3.4. Recall that $W_{a}^{R}(x)=\inf \left\{W^{R}(y)|| x-y \mid \leq a\right\}$. Then

$$
\lim _{|x| \rightarrow \infty} W_{|x| / 2}^{|x|}(x)=0 .
$$

Hence there exists a positive constant $R^{*}$ such that $\left|W_{|X| / 2}^{|X|}(X)\right| \leq|\tilde{E}| / 2$ for all $X$ such that $|X|>R^{*}$. Suppose that $|X|>R^{*}$ and let $R=|X|$. We divide $W_{\text {eff }}$ as in (36) for $R$. We have

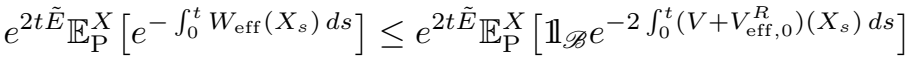

$$
\begin{aligned}
& +e^{2 t \tilde{E}}\left(\mathbb{E}_{\mathrm{P}}^{X}\left[\mathbb{1}_{\mathscr{B} c} e^{-4 \int_{0}^{t}\left(V+V_{\text {eff }, 0}^{R}\right)\left(X_{s}\right) d s}\right]\right)^{1 / 2}\left(\mathbb{E}_{\mathrm{P}}^{X}\left[\mathbb{1}_{\mathscr{B} c} e^{-4 \int_{0}^{t}\left(V+V_{\text {eff }, \infty}^{R}\right)\left(X_{s}\right) d s}\right]\right)^{1 / 2} .
\end{aligned}
$$

Now,

$$
\begin{aligned}
& \mathbb{E}_{\mathrm{P}}^{X}\left[\mathbb{1}_{\mathscr{B}} e^{-2 \int_{0}^{t}\left(V+V_{\mathrm{eff}, 0}^{R}\right)\left(X_{s}\right) d s}\right] \leq e^{-2 t W_{a}^{R}(x)}+C_{1} e^{-2 t W_{\infty}^{R} e^{C_{2} t} e^{-\eta a},} \\
& \mathbb{E}_{\mathrm{P}}^{x}\left[\mathbb{1}_{\mathscr{B} c} e^{-4 \int_{0}^{t}\left(V+V_{\mathrm{eff}, 0}^{R}\right)\left(X_{s}\right) d s}\right] \leq e^{-4 t W_{a}^{R}(x)}+C_{1} e^{-4 t W_{\infty}^{R} e^{C_{2} t} e^{-\eta a},}
\end{aligned}
$$

by Lemma 3.6. Set $t=t(X)=\epsilon|X|$ and $a=|X| / 2$. Then $W_{|X| / 2}^{|X|}(X)-\tilde{E}>$ $-\tilde{E} / 2>0$, since $\tilde{E}<0$ by assumption. Hence

$$
\begin{aligned}
& e^{2 t \tilde{E}} \mathbb{E}_{\mathrm{P}}^{X}\left[\mathbb{1}_{\mathscr{B}} e^{-2 \int_{0}^{t}\left(V+V_{\mathrm{eff}, 0}^{R}\right)\left(X_{s}\right) d s}\right] \leq e^{\epsilon \tilde{E}|X|}+C_{2} e^{\epsilon C_{2}|X|-\eta|X| / 2-2 \epsilon W_{\infty}^{|X|}|X|} \\
& \leq e^{-\epsilon \gamma|X|}+C_{2} e^{-\left(\eta / 2+2 \epsilon W_{\infty}^{|X|}-\epsilon C_{2}\right)|X|} \text {. }
\end{aligned}
$$

Similarly,

$$
e^{4 t \tilde{E}} \mathbb{E}_{\mathrm{P}}^{X}\left[\mathbb{1}_{\mathscr{B}} e^{-4 \int_{0}^{t}\left(V+V_{\text {eff }, 0}^{R}\right)\left(X_{s}\right) d s}\right] \leq e^{-2 \epsilon \gamma|X|}+C_{2} e^{-\left(\eta / 2+4 \epsilon W_{\infty}^{|X|}-\epsilon C_{2}\right)|X|} .
$$

Finally, by Lemma 3.7 we have

$$
\begin{aligned}
e^{4 t \tilde{E}} \mathbb{E}_{\mathrm{P}}^{X}\left[\mathbb{1}_{\mathscr{B}} e^{-4 \int_{0}^{t} W_{\mathrm{eff}}\left(X_{s}\right) d s}\right] & \leq C_{1} e^{\left.4 \epsilon \tilde{E}+4\left\|V_{\text {eff }, \infty}\right\|_{\infty} \epsilon+C_{2} \epsilon-\eta\right)|X|} \\
& \leq C_{1} e^{-\left(4 \epsilon \gamma-4\left\|V_{\text {eff }, \infty}\right\|_{\infty} \epsilon-C_{2} \epsilon+\eta\right)|X|}
\end{aligned}
$$

Note that $W_{\infty}^{|X|} \rightarrow 0$ as $|X| \rightarrow \infty$. Take a sufficiently small $\epsilon>0$ such that $\eta / 2+\left(2 W_{\infty}^{|X|}-C_{2}\right) \epsilon>0, \eta / 2+\left(4 W_{\infty}^{|X|}-C_{2}\right) \epsilon>0$ and $\left(4 \gamma-4\left\|V_{\text {eff }, \infty}\right\|_{\infty}-C_{2}\right) \epsilon+\eta$ $>0$. Then $\left\|\Phi_{\sigma}(X)\right\|_{\mathscr{F}} \leq D_{1} e^{-\min \{\eta, \gamma\} D_{2}|X|}$ follows.

Corollary 3.9. Suppose (EN)(1) holds. Then (44) holds for sufficiently small $|\alpha / \kappa|$.

Proof. Notice that $E^{V} \leq \mathcal{E}^{V}+\mathcal{G}(\alpha / \kappa)-E_{\text {diag }}$ by Lemma 3.3. Since $\mathcal{E}^{V}<0$ and $\lim _{t \rightarrow 0} \mathcal{G}(t)=0$, the corollary follows. 


\section{§3.3. Proofs of Theorems 2.2 and 2.3}

3.3.1. Proof of Theorem 2.2. Note that $0<\mathcal{E}^{V}-\Xi^{V}$ is equivalent to inf $\sigma\left(H_{\text {eff }}^{V}\right)$ $\in \sigma_{\text {disc }}\left(H_{\text {eff }}^{V}\right)$. The uniform exponential decay

$$
\left\|\Phi_{\sigma}(x)\right\|_{\mathscr{F}} \leq C_{\delta} e^{-\delta|x|}
$$

is shown for sufficiently small $|\alpha / \kappa|$ in Lemma 3.8. Then from $\lim _{\kappa \rightarrow \infty} \mathcal{G}(\alpha / \kappa)=0$ and $\Sigma^{V}-E^{V} \geq \Xi^{V}-\mathcal{E}^{V}-\mathcal{G}(\alpha / \kappa)$, there exists $\kappa_{0}$ such that for all $\kappa>\kappa_{0}$ the stability condition $E^{V}<\Sigma^{V}$ holds. Therefore, by Proposition 2.7, $H^{V}$ has a ground state.

3.3.2. Proof of Theorem 2.3. Now we prove enhanced binding. By Proposition 2.7 it is enough to show $\mathcal{E}^{V}<\Xi^{V}$, since the uniform exponential decay $\left\|\Phi_{\sigma}(x)\right\|_{\mathscr{F}}<C_{\delta} e^{-\delta|x|}$ is already established.

Lemma 3.10. Let $\beta \subsetneq C_{N}$ but $\beta \neq \emptyset$. Then there exists $\alpha_{1}>0$ such that, for all $\alpha$ with $|\alpha|>\alpha_{1}, \mathcal{E}^{0}<\mathcal{E}^{V}(\beta)+\mathcal{E}^{0}\left(\beta^{c}\right)$. In particular $\mathcal{E}^{0}<\Xi^{V}$ for $|\alpha|>\alpha_{1}$.

Proof. We have

$$
\begin{aligned}
& \mathcal{E}^{0}=\alpha^{2} \sum_{i<j} W_{i j}(0)+o\left(\alpha^{2}\right), \quad \mathcal{E}^{V}(\beta)=\alpha^{2} \sum_{\substack{i<j \\
i, j \in \beta}} W_{i j}(0)+o\left(\alpha^{2}\right), \\
& \mathcal{E}^{0}\left(\beta^{c}\right)=\alpha^{2} \sum_{\substack{i<j \\
i, j \in \beta^{c}}} W_{i j}(0)+o\left(\alpha^{2}\right) .
\end{aligned}
$$

Since

the lemma holds.

$$
\sum_{\substack{i<j \\ i \in \beta, j \in \beta^{c}}} W_{i j}(0)+\sum_{\substack{i<j \\ i \in \beta^{c}, j \in \beta}} W_{i j}(0)<0,
$$

To see enhanced binding we want to investigate the center of motion of $h_{\text {eff }}^{V}$. Notice that $h_{\text {eff }}^{0}$ commutes with the total momentum $P_{\text {tot }}=\sum_{j=1}^{N} p_{j}$, so it can be decomposed with respect to the spectrum of $P_{\text {tot }}$. Let $\mathscr{U}=e^{i x_{1} \cdot \sum_{j=2}^{N} p_{j}}$, which diagonalizes $P_{\text {tot }}$ as $\mathscr{U} P_{\text {tot }} \mathscr{U}^{-1}=p_{1}$. Hence it also diagonalizes $h_{\text {eff }}^{0}$, and we obtain

$$
\begin{aligned}
\mathscr{U} h_{\mathrm{eff}}^{0} \mathscr{U}^{-1}= & \Omega_{1}\left(p_{1}-\sum_{j=2}^{N} p_{j}\right)+\sum_{j=2}^{N} \Omega_{j}\left(p_{j}\right)+\sum_{j \geq 2} \alpha^{2} W_{1 j}\left(x_{j}\right) \\
& +\sum_{2 \leq i<j \leq N} \alpha^{2} W_{i j}\left(x_{i}-x_{j}\right), \\
\mathscr{U} h_{\mathrm{eff}}^{V} \mathscr{U}^{-1}= & h_{\mathrm{eff}}^{0}+V\left(x_{1}\right)+\sum_{j=2}^{N} V\left(x_{1}+x_{j}\right) .
\end{aligned}
$$


Consequently,

$$
\begin{aligned}
& \mathscr{U} h_{\mathrm{eff}}^{0} \mathscr{U}^{-1}=\int_{\mathbb{R}^{d}}^{\oplus} k(P) d P, \\
& k(P)=\Omega_{1}\left(P-\sum_{j=2}^{N} p_{j}\right)+\sum_{j=2}^{N} \Omega_{j}\left(p_{j}\right)+\sum_{j \geq 2} \alpha^{2} W_{1 j}\left(x_{j}\right)+\sum_{2 \leq i<j \leq N} \alpha^{2} W_{i j}\left(x_{i}-x_{j}\right) .
\end{aligned}
$$

Lemma 3.11. We have $\mathcal{E}^{0}=\inf \sigma(k(0))$.

Proof. Set inf $\sigma(k(P))=E(P)$ for notational simplicity. It can be seen in Appendix 7 that

$$
E(0) \leq E(P)
$$

for all $P$, and $E(P)$ is continuous in $P$. Hence $(\Phi, H \Phi)=\int_{\mathbb{R}^{d}}(\Phi(P), k(P) \Phi(P)) d P$ $\geq E(0)\|\Phi\|^{2}$ for $\Phi \in D(H)$. Thus $E(0) \leq \mathcal{E}^{0}$. On the other hand, set $\Phi_{\epsilon}=$ $\int_{\mathbb{R}^{d}}^{\oplus} \Phi(P) \mathbb{1}_{[0, \epsilon)}(P) d P$. Then

$$
\left\|\Phi_{\epsilon}\right\|^{2} \mathcal{E}^{0} \leq\left(\Phi_{\epsilon}, H \Phi_{\epsilon}\right) \leq \sup _{|P|<\epsilon} E(P)\left\|\Phi_{\epsilon}\right\|^{2} .
$$

Taking $\epsilon \downarrow 0$ on both sides we get $\mathcal{E}^{0} \leq E(0)+\delta$ for all $\delta>0$, since $E(P)$ is continuous in $P$. Hence $E(0) \geq \mathcal{E}^{0}$, and $\mathcal{E}^{0}=E(0)$ follows.

Lemma 3.12. There exists $\alpha_{2}(P)>0$ such that inf $\sigma(k(P)) \in \sigma_{\text {disc }}(k(P))$ for every $P \in \mathbb{R}^{d}$ for $|\alpha|>\alpha_{2}(P)$. In particular $k(0)$ has a ground state for $|\alpha|>\alpha_{2}$ with some $\alpha_{2}>0$.

Proof. Note that $W_{i j}(0)<0, W_{i j}(x)>W_{i j}(0)$ for $x \neq 0$, and $\lim _{|x| \rightarrow \infty} W_{i j}(x)=0$. Set $\mathbf{X}=\left(x_{2}, \ldots, x_{N}\right)$. Let $a=\{2, \ldots, N\}$. Let $\left\{\widetilde{j}_{\beta}\right\}_{\beta \subset a}$ be the Ruelle-Simon partition of unity [CFKS87, Definition 3.4], i.e., $\widetilde{j}_{\beta}(\lambda \mathbf{X})=\widetilde{j}_{\beta}(\mathbf{X})$ for all $\lambda>1$, $|\mathbf{X}|=1$, and there exists a constant $C>0$ such that

$$
\operatorname{supp} \widetilde{j}_{\beta} \cap\{\mathbf{X}|| \mathbf{X} \mid>1\} \subset\left\{\mathbf{X}|| \mathbf{X}_{i}-\mathbf{X}_{j}|\geq C| \mathbf{X} \mid \text { for all }\{i, j\} \not \subset \beta\right\} \text {. }
$$

We set $j_{\beta}(\mathbf{X})=\widetilde{j}_{\beta}(\mathbf{X} / R)$. Then

$$
k(P)=j_{a} k(P) j_{a}+\sum_{\beta \subsetneq a} j_{\beta} k(P) j_{\beta}+o(\mathbb{1}),
$$

where $o(\mathbb{1})$ denotes a bounded operator such that $\lim _{R \rightarrow \infty}\|o(\mathbb{1})\|=0$. We set

$$
\begin{aligned}
& k_{\beta}=\sum_{j \in \beta}\left(\Omega_{j}\left(p_{j}\right)+\alpha^{2} W_{1 j}\left(x_{j}\right)\right)+\sum_{i, j \in \beta} \alpha^{2} W_{i j}\left(x_{i}-x_{j}\right), \\
& \bar{k}_{\beta^{c}}=\sum_{j \in \beta^{c}} \Omega_{j}\left(p_{j}\right)+\sum_{i, j \in \beta^{c}} \alpha^{2} W_{i j}\left(x_{i}-x_{j}\right) .
\end{aligned}
$$


With the identification $L^{2}\left(\mathbb{R}^{d(N-1)}\right) \cong L^{2}\left(\mathbb{R}^{d|\beta|}\right) \otimes L^{2}\left(\mathbb{R}^{\left.d\left|\beta^{c}\right|\right)}\right)$, we can write

$$
j_{\beta} k(P) j_{\beta}=j_{\beta} \Omega_{1}\left(P-\sum_{j=2}^{N} p_{j}\right) j_{\beta}+j_{\beta}\left(k_{\beta} \otimes \mathbb{1}+\mathbb{1} \otimes \bar{k}_{\beta^{c}}\right) j_{\beta}+I_{\beta} j_{\beta}^{2}
$$

where $I_{\beta}=\sum_{j \in \beta^{c}} \alpha^{2} W_{1 j}\left(x_{j}\right)+\sum_{\substack{i \in \beta, j \in \beta^{c} \\ i \in \beta^{c}, j \in \beta}} \alpha^{2} W_{i j}\left(x_{i}-x_{j}\right)$. Hence, (49) and (50) imply

$$
k(P) \geq E_{0}(k(P)) j_{a}^{2}+\sum_{\beta \subsetneq a} j_{\beta}\left(k_{\beta} \otimes \mathbb{1}+\mathbb{1} \otimes \bar{k}_{\beta^{c}}+I_{\beta}\right) j_{\beta}+o(\mathbb{1}) .
$$

Note that $j_{a}^{2}$ and $I_{\beta} j_{\beta}^{2}$ are relatively compact with respect to $k(P)$. Thus we have

$$
\inf \sigma_{\text {ess }}(k(P)) \geq \max \left\{E_{0}\left(k_{\beta}\right)+E_{0}\left(\bar{k}_{\beta^{c}}\right) \mid \beta \subsetneq a\right\} .
$$

For all $\beta \subsetneq a$,

$$
\text { (51) } \begin{aligned}
\lim _{\alpha \rightarrow \infty} \frac{E_{0}(k(P))}{\alpha^{2}} & =\sum_{i<j} W_{i j}(0)<\sum_{j \in \beta} W_{1 j}(0)+\sum_{\substack{i, j \in \beta \\
i<j}} W_{i j}(0)+\sum_{\substack{i, j \in \beta^{c} \\
i<j}} W_{i j}(0) \\
& =\lim _{\alpha \rightarrow \infty} \frac{E_{0}\left(k_{\beta}\right)+E_{0}\left(\bar{k}_{\beta^{c}}\right)}{\alpha^{2}} .
\end{aligned}
$$

Therefore there exist $\alpha_{2}(P)$ such that inf $\sigma_{\text {eff }}(k(P))>E_{0}(k(P))$ for all $\alpha>\alpha_{2}(P)$.

Lemma 3.13. Let $|\alpha|>\alpha_{2}$, where $\alpha_{2}$ is as in Lemma 3.12, and $u_{\alpha}$ be a normalized ground state of $k(0)$. Then $\left|u_{\alpha}\left(x_{2}, \ldots, x_{N}\right)\right|^{2} \rightarrow \delta\left(x_{2}\right) \cdots \delta\left(x_{N}\right)$ as $\alpha \rightarrow \infty$ in the sense of distributions.

Proof. It suffices to show that for all $\epsilon>0$,

$$
\lim _{\alpha \rightarrow \infty} \int_{|\mathbf{X}|>\epsilon}\left|u_{\alpha}(\mathbf{X})\right|^{2} d \mathbf{X}=0
$$

where $\mathbf{X}=\left(x_{2}, \ldots, x_{N}\right)$, since (52) implies that

$$
\lim _{\alpha \rightarrow 0} \int_{\mathbb{R}^{d(N-1)}} f(\mathbf{X})\left|u_{\alpha}(\mathbf{X})\right|^{2} d \mathbf{X}=f(0)
$$

for all $f \in C_{0}^{\infty}\left(\mathbb{R}^{d(N-1)}\right)$. We write $k_{\alpha}(0)$ to emphasize the $\alpha$ dependence of $k(0)$. Since $k_{\alpha}(0) / \alpha^{2} \geq \sum_{i<j} W_{i j}(0)$ and $\lim _{\alpha \rightarrow \infty} \inf \sigma\left(k_{\alpha}(0)\right) / \alpha^{2}=\sum_{i<j} W_{i j}(0)$, we 
have

$$
\begin{aligned}
& \sum_{i<j} W_{i j}(0)=\lim _{\alpha \rightarrow 0} \alpha^{-2}\left(u_{\alpha}, k_{\alpha}(0) u_{\alpha}\right) \\
& \geq \liminf _{\alpha \rightarrow \infty}\left(u_{\alpha},\left(\sum_{j \geq 2} W_{1 j}\left(x_{j}\right)+\sum_{2 \leq i<j \leq N} W_{i j}\left(x_{i}-x_{j}\right)\right) u_{\alpha}\right) \geq \sum_{i<j} W_{i j}(0) .
\end{aligned}
$$

Thus

$$
\sum_{i<j} W_{i j}(0)=\liminf _{\alpha \rightarrow \infty}\left(u_{\alpha},\left(\sum_{j \geq 2} W_{1 j}\left(x_{j}\right)+\sum_{2 \leq i<j \leq N} W_{i j}\left(x_{i}-x_{j}\right)\right) u_{\alpha}\right) .
$$

Suppose that $c_{\epsilon}=\liminf _{\alpha \rightarrow \infty} \int_{|\mathbf{X}|>\epsilon}\left|u_{\alpha}(\mathbf{X})\right|^{2} d \mathbf{X}>0$. Then

$$
\begin{aligned}
\liminf _{\alpha \rightarrow \infty} \int_{\mathbb{R}^{d(N-1)}} \sum_{j \geq 2}\left(W_{1 j}\left(x_{j}\right)-W_{1 j}(0)\right)\left|u_{\alpha}(\mathbf{X})\right|^{2} d \mathbf{X} \\
\quad>c_{\epsilon} \sum_{j \geq 2} \sup _{|\mathbf{X}|>\epsilon}\left(W_{1 j}\left(x_{j}\right)-W_{1 j}(0)\right)>0,
\end{aligned}
$$

which contradicts (53). Therefore (52) holds.

Proof of Theorem 2.3. First we assume that $V \in C_{0}^{\infty}\left(\mathbb{R}^{d}\right)$. It is enough to show $\mathcal{E}^{V}<\Xi^{V}$, since the uniform exponential decay $\left\|\Phi_{\sigma}(x)\right\| \leq C_{\delta} e^{-\delta|x|}$ is established in Lemma 3.8 for sufficiently small $|\alpha / \kappa|$. Assume $|\alpha|>\max \left\{\alpha_{1}, \alpha_{2}\right\}>0$. Let $u_{\alpha}$ be a normalized ground state of $k(0)$. From $\Omega_{1}(a+b) \leq|a|+\Omega_{1}(b)$ for $a, b \in \mathbb{R}^{d}$, we have

$$
\mathscr{U} h_{\mathrm{eff}}^{0} \mathscr{U}^{-1} \leq \sqrt{-\Delta_{1}}+k(0)
$$

By $(\mathrm{EN})(2)$, there exists a normalized vector $v \in C_{0}^{\infty}\left(\mathbb{R}^{d}\right)$ such that

$$
(v,(\sqrt{-\Delta}+N V) v)<0 .
$$

We set $\Psi\left(x_{1}, \ldots, x_{N}\right)=v\left(x_{1}\right) u_{\alpha}\left(x_{2}, \ldots, x_{N}\right)$. Then, by $(54)$,

(56) $\mathcal{E}^{V} \leq\left(\Psi, \mathscr{U} h_{\mathrm{eff}}^{V} \mathscr{U}^{-1} \Psi\right) \leq(v,(\sqrt{-\Delta}+V) v)+\mathcal{E}^{0}+\left(\Psi, \sum_{j=2}^{N} V\left(x_{1}+x_{j}\right) \Psi\right)$.

Let $V_{j, \text { smeared }}^{\alpha}\left(x_{1}\right)=\int_{\mathbb{R}^{d(N-1)}} V\left(x_{j}+x_{1}\right)\left|u_{\alpha}(\mathbf{X})\right|^{2} d \mathbf{X}$. By Lemma 3.13,

$$
\lim _{\alpha \rightarrow \infty}\left(\Psi, V\left(x_{j}+x_{1}\right) \Psi\right)=\lim _{\alpha \rightarrow \infty}\left(v, V_{j, \text { smeared }}^{\alpha} v\right)=(v, V v),
$$

and so by (55) and (56),

$$
\mathcal{E}^{V} \leq(v,(\sqrt{-\Delta}+N V) v)+\mathcal{E}^{0}<\mathcal{E}^{0}
$$


for $\alpha>\alpha_{3}$ with some $\alpha_{3}>0$. From this inequality and Lemma 3.10, we conclude that for $\alpha$ with $|\alpha|>\bar{\alpha}=\max \left\{\alpha_{1}, \alpha_{2}, \alpha_{3}\right\}$,

$$
\begin{aligned}
\Sigma^{V}-E^{V} & \geq \Xi^{V}-\mathcal{E}^{V}-\mathcal{G}(\alpha / \kappa) \geq \mathcal{E}^{0}-\mathcal{E}^{V}-\mathcal{G}(\alpha / \kappa) \\
& >-(v,(\sqrt{-\Delta}+N V) v)-\mathcal{G}(\alpha / \kappa) .
\end{aligned}
$$

Notice that $\mathcal{G}(\alpha / \kappa) \rightarrow 0$ as $\kappa \rightarrow \infty$, and $-(v,(\sqrt{-\Delta}+N V) v)>0$. Thus the right-hand side above is positive for sufficiently small $|\alpha| / \kappa$. Since $\mathcal{G}$ is increasing, it is trivial to see that $\kappa_{0}=\bar{\alpha} / \mathcal{G}^{-1}(a)$, where $a=-(v,(\sqrt{-\Delta}+N V) v)$ and $\bar{\alpha}(\kappa)=\mathcal{G}^{-1}(a) \kappa$. Thus the theorem follows for $V \in C_{0}^{\infty}\left(\mathbb{R}^{d}\right)$. For general $V$ we can use the limiting argument of [HS08, Appendix]. See Appendix 5.

\section{Appendix}

\section{$\S 4$. Stability condition: relativistic version}

In this section we shall prove Proposition 2.7. We only give an outline of the proof. The details are left to the reader.

\section{§4.1. Case $\sigma>0$}

Since the scaling parameter $\kappa$ does not play any role in this section, we set $\kappa=1$. Let $\sigma>0$. We decompose the single boson Hilbert space into high energy and low energy parts, $L^{2}\left(\mathbb{R}^{d}\right) \cong \mathcal{K}_{>\sigma} \oplus \mathcal{K}_{\leq \sigma}$, where $\mathcal{K}_{\leq \sigma}=L^{2}\left(\left\{k \in \mathbb{R}^{d} \mid \omega(k) \leq \sigma\right\}\right)$ and $\mathcal{K}_{>\sigma}=L^{2}\left(\left\{k \in \mathbb{R}^{d} \mid \omega(k)>\sigma\right\}\right)$. Correspondingly, we have the identification

$$
\mathscr{H} \cong \mathscr{H}_{>\sigma} \otimes \mathscr{F}\left(\mathcal{K}_{\leq \sigma}\right),
$$

where $\mathscr{H}_{>\sigma}=L^{2}\left(\mathbb{R}^{d N}\right) \otimes \mathscr{F}\left(\mathcal{K}_{>\sigma}\right)$. We define the regularized Hamiltonian by

$$
H_{\sigma}^{V}=H_{0}+H_{\mathrm{I}, \sigma}
$$

Here $H_{\mathrm{I}, \sigma}$ is the regularized interaction defined by

$$
H_{\mathrm{I}, \sigma}=\sum_{j=1}^{N} \alpha_{j} \int_{\mathbb{R}^{d N}}^{\oplus} \phi_{j, \sigma}\left(x_{j}\right) d X,
$$

and $\phi_{j, \sigma}(x)$ is $\phi_{j}(x)$ with cutoff $\lambda_{j}(k)$ replaced by $\lambda_{j}(k) \mathbb{1}_{\omega(k)>\sigma}(k)$. Then $H_{\sigma}^{V}$ approximates $H^{V}$ in the following sense:

Lemma 4.1. $H_{\sigma}^{V}$ converges to $H^{V}$ as $\sigma \rightarrow 0$ in the norm resolvent sense.

Let $E_{\sigma}^{V}=\inf \sigma\left(H_{\sigma}^{V}\right)$ and let $\Sigma_{\sigma}^{V}$ be the lowest two-cluster threshold for $H_{\sigma}^{V}$, defined in the same way as $\Sigma^{V}$. From Lemma 4.1, we can show that $E_{\sigma}^{V}$ and $\Sigma_{\sigma}^{V}$ 
converge to $E^{V}$ and $\Sigma^{V}$ respectively as $\sigma \rightarrow 0$. Therefore for sufficiently small $\sigma>0$

$$
\Sigma_{\sigma}^{V}>E_{\sigma}^{V} .
$$

Under the identification (58), $H_{\sigma}^{V}$ can be decomposed as

$$
H_{\sigma}^{V} \cong H_{\sigma}^{V}\left\lceil_{\mathscr{H}_{>\sigma}} \otimes \mathbb{1}_{\mathscr{F}\left(\mathcal{K}_{\leq \sigma}\right)}+\mathbb{1}_{\mathscr{H}_{>\sigma}} \otimes H_{\mathrm{f}}\left\lceil_{\mathscr{F}\left(\mathcal{K}_{\leq \sigma}\right)} .\right.\right.
$$

Since $H_{\mathrm{f}}\left\lceil\mathscr{F}\left(\mathcal{K}_{<\sigma}\right)\right.$ has a ground state, $H_{\sigma}^{V}$ may have a ground state if and only if $H_{\sigma}^{V}\left\lceil_{\mathscr{H}_{>\sigma}}\right.$ does. We shall prove the existence of a ground state of $H_{\sigma}^{V}\left\lceil\mathscr{H}_{>\sigma}\right.$ for sufficiently small $\sigma>0$. For $\sigma>0$, we truncate $\omega$ as

$$
\omega_{\sigma}(k)= \begin{cases}|k| & \text { for }|k|>\sigma, \\ \sigma & \text { for }|k| \leq \sigma,\end{cases}
$$

and we set $H_{\mathrm{f}, \sigma}=\mathrm{d} \Gamma\left(\omega_{\sigma}\right)$. Then

$$
H_{\sigma}^{V}\left\lceil_{\mathscr{H}_{>\sigma}}=H_{0, \sigma}+H_{\mathrm{I}, \sigma}\right.
$$

with $H_{0, \sigma}=H_{\mathrm{p}} \otimes \mathbb{1}+\mathbb{1} \otimes H_{\mathrm{f}, \sigma}$. We denote the Fourier transformation from $L^{2}\left(\mathbb{R}_{y}^{d}\right)$ to $L^{2}\left(\mathbb{R}_{k}^{d}\right)$ by $F$. We set $\check{\mathcal{K}}_{>\sigma}=\left\{\check{f}=F^{-1} f \in L^{2}\left(\mathbb{R}_{y}^{d}\right) \mid f \in \mathcal{K}_{>\sigma}\right\}$. We introduce some notation. Let $T: \mathcal{K}_{1} \rightarrow \mathcal{K}_{2}$ be a contraction operator from a Hilbert space $\mathcal{K}_{1}$ to another one $\mathcal{K}_{2}$. Then we define $\Gamma(T)=\bigoplus_{n=0}^{\infty} \otimes^{n} T$ with $\otimes^{0} T=\mathbb{1}$, which is also a contraction operator from $\mathscr{F}\left(\mathcal{K}_{1}\right)$ to $\mathscr{F}\left(\mathcal{K}_{2}\right)$. Let

$$
\check{H}_{\sigma}^{V}=\Gamma\left(F^{-1}\right) H_{\sigma}^{V}\left\lceil\mathscr{H}_{>\sigma} \Gamma(F),\right.
$$

which is defined on $\check{\mathscr{H}}_{>\sigma}=L^{2}\left(\mathbb{R}^{d N}\right) \otimes \mathscr{F}\left(\check{\mathcal{K}}_{>\sigma}\right)$. Let $\chi, \bar{\chi} \in C^{\infty}\left(\mathbb{R}^{d N}\right)$ be cutoff functions such that $\chi(X)^{2}+\bar{\chi}(X)^{2}=1$ with $\chi(X)=1$ for $|X| \leq 1$ and $\chi(X)=0$ for $|X| \geq 2$. For $R>0$, we set $\chi_{R}(X)=\chi(X / R), \bar{\chi}_{R}(X)=\bar{\chi}(X / R)$.

Lemma 4.2. We have

$$
\check{H}_{\sigma}^{V}=\chi_{R} \check{H}_{\sigma}^{V} \chi_{R}+\bar{\chi}_{R} \check{H}_{\sigma}^{V} \bar{\chi}_{R}+\hat{O}\left(R^{-1}\right),
$$

where $\hat{O}\left(R^{-1}\right)$ is an operator such that $\left\|\hat{O}\left(R^{-1}\right)\right\| \leq C / R$ for some constant $C>0$.

Proof. We have the operator equality

$$
\begin{aligned}
\check{H}_{\sigma}^{V}= & \chi_{R} \check{H}_{\sigma}^{V} \chi_{R}+\bar{\chi}_{R} \check{H}_{\sigma}^{V} \bar{\chi}_{R} \\
& +\frac{1}{2} \sum_{j=1}^{N}\left[\chi_{R},\left[\chi_{R}, \Omega_{j}\left(p_{j}\right)\right]\right]+\frac{1}{2} \sum_{j=1}^{N}\left[\bar{\chi}_{R},\left[\bar{\chi}_{R}, \Omega_{j}\left(p_{j}\right)\right]\right] .
\end{aligned}
$$


By the Fourier transformation,

$$
\left[\chi_{R}, \Omega_{j}\left(p_{j}\right)\right]=(2 \pi)^{-d N / 2} \int_{\mathbb{R}^{d N}} \hat{\chi}(K) e^{i K \cdot X / R}\left(\Omega_{j}\left(p_{j}\right)-\Omega_{j}\left(p_{j}-k_{j} / R\right)\right) d K,
$$

where $K=\left(k_{1}, \ldots, k_{N}\right) \in \mathbb{R}^{d N}$. By the triangle inequality,

$$
\begin{aligned}
\left|\Omega_{j}\left(p_{j}\right)-\Omega_{j}\left(p_{j}-k_{j} / R\right)\right| & =\left|\left\|\left(p_{j}, m_{j}\right)\right\|_{\mathbb{C}^{4}}-\left\|\left(p_{j}-k_{j} / R, m_{j}\right)\right\|_{\mathbb{C}^{4}}\right| \\
& \leq\left\|\left(k_{j} / R, 0\right)\right\|_{\mathbb{C}^{4}}=\frac{1}{R}\left|k_{j}\right| .
\end{aligned}
$$

Hence, $\left[\chi_{R}, \Omega_{j}\left(p_{j}\right)\right]$ is a bounded operator with

$$
\left\|\left[\chi_{R}, \Omega_{j}\left(p_{j}\right)\right]\right\| \leq \frac{1}{R}(2 \pi)^{-d N / 2} \int_{\mathbb{R}^{d N}}|\hat{\chi}(K)| \cdot\left|k_{j}\right| d K
$$

Similarly, as $\mathbb{1}-\bar{\chi} \in C_{0}^{\infty}\left(\mathbb{R}^{d N}\right)$ and $\left[\bar{\chi}_{R}, \Omega_{j}\left(p_{j}\right)\right]=\left[\mathbb{1}-\bar{\chi}_{R}, \Omega_{j}\left(p_{j}\right)\right]$, we have

$$
\left.\left\|\left[\bar{\chi}_{R}, \Omega_{j}\left(p_{j}\right)\right]\right\| \leq \frac{1}{R}(2 \pi)^{-d N / 2} \int_{\mathbb{R}^{d N}} \mid \widehat{11} \overline{-\bar{\chi}(K}\right)|\cdot| k_{j} \mid d K .
$$

Hence the lemma follows.

Let $j, \bar{j} \in C_{0}^{\infty}\left(\mathbb{R}^{d}\right)$ be another pair of cutoff functions such that $j(y)^{2}+\bar{j}(y)^{2}$ $=1$ for every $y \in \mathbb{R}^{d}$ with $j(y)=1$ for $|y| \leq 1$ and $j(y)=0$ for $|y| \geq 2$. We set $j_{P}(y)=j(y / P)$ and $\bar{j}_{P}(y)=\bar{j}(y / P)$ for $P>0$. The map

$$
u_{P}: \check{\mathcal{K}}_{>\sigma} \rightarrow L^{2}\left(\mathbb{R}_{y}^{d}\right) \oplus L^{2}\left(\mathbb{R}_{y}^{d}\right), \quad f \mapsto j_{P} f \oplus \bar{j}_{P} f,
$$

is an isometry, since $\left\|j_{P} f \oplus \bar{j}_{P} f\right\|^{2}=\|f\|^{2}$. We also note that the adjoint $u_{P}^{*}$ maps $f \oplus g \in L^{2}\left(\mathbb{R}_{y}^{d}\right) \oplus L^{2}\left(\mathbb{R}_{y}^{d}\right)$ to $j_{P} f+\bar{j}_{P} g \in L^{2}\left(\mathbb{R}^{d}\right)$. The operator

$$
U_{P}=\mathbb{1}_{L^{2}\left(\mathbb{R}^{d N}\right)} \otimes \Gamma\left(u_{P}\right): \check{\mathscr{H}}_{>\sigma} \rightarrow \check{\mathscr{H}} \otimes \mathscr{F}\left(L^{2}\left(\mathbb{R}_{y}^{d}\right)\right)
$$

is also an isometry, where $\check{\mathscr{H}}=L^{2}\left(\mathbb{R}^{d N}\right) \otimes \mathscr{F}\left(L^{2}\left(\mathbb{R}_{y}^{d}\right)\right)$. Let

$$
\check{H}_{0, \sigma}=\Gamma\left(F^{-1}\right) H_{0, \sigma} \Gamma(F), \quad \check{H}_{\mathrm{f}, \sigma}=\Gamma\left(F^{-1}\right) H_{\mathrm{f}, \sigma} \Gamma(F) .
$$

Lemma 4.3. (1) For every $\sigma>0$, we have

$$
\chi_{R} \check{H}_{\sigma}^{V} \chi_{R}=\chi_{R} U_{P}^{*}\left\{\check{H}_{\sigma}^{V} \otimes \mathbb{1}+\mathbb{1} \otimes \check{H}_{\mathrm{f}, \sigma}\right\} U_{P} \chi_{R}+\hat{o}(\mathbb{1})
$$

as operators in $\mathscr{H}_{>\sigma}$, where $\hat{o}(\mathbb{1})$ is an operator such that $\hat{o}(\mathbb{1})\left(\check{H}_{0, \sigma}+\mathbb{1}\right)^{-1}$ is bounded and $\lim _{P \rightarrow \infty} \lim _{R \rightarrow \infty}\left\|\hat{o}(\mathbb{1})\left(\check{H}_{0, \sigma}+1\right)^{-1}\right\|=0$.

(2) We have

$$
\bar{\chi}_{R} \check{H}_{\sigma}^{V} \bar{\chi}_{R} \geq \Sigma_{\sigma}^{V} \bar{\chi}_{R}^{2}+o\left(R^{0}\right),
$$

where $o\left(R^{0}\right)$ is a number such that $\lim _{R \rightarrow \infty} o\left(R^{0}\right)=0$.

Proof. See [GLL01, Lemma A.1]. 
Proposition 4.4. There exists a ground state of $H_{\sigma}^{V}$.

Proof. By Lemmas 4.2 and 4.3,

$$
\check{H}_{\sigma}^{V}=\chi_{R} U_{P}^{*}\left\{\check{H}_{\sigma}^{V} \otimes \mathbb{1}+\mathbb{1} \otimes \check{H}_{\mathrm{f}, \sigma}\right\} U_{P} \chi_{R}+\bar{\chi}_{R} \check{H}_{\sigma}^{V} \bar{\chi}_{R}+\hat{o}(\mathbb{1}) .
$$

Since $\omega_{\sigma} \geq \sigma$, we have $\check{H}_{\mathrm{f}, \sigma} \geq \sigma\left(1-P_{\Omega}\right)$, where $P_{\Omega}$ denotes the orthogonal projection on the vacuum space $\{\mathbb{C} \Omega\}$. By this inequality and Lemma 4.3,

$$
\check{H}_{\sigma}^{V} \geq\left(E_{\sigma}^{V}+\sigma\right) \chi_{R}^{2}+\Sigma_{\sigma}^{V} \bar{\chi}_{R}^{2}-K+\hat{o}(1),
$$

where $K=\sigma \chi_{R} U_{P}^{-1}\left(\mathbb{1} \otimes P_{\Omega}\right) U_{P} \chi_{R}=\chi_{R}^{2} \otimes \Gamma\left(j_{P}\right)$. Here $K$ is relatively compact with respect to $\sum_{j=1}^{N} \Omega_{j}+\check{H}_{\mathrm{f}, \sigma}$. Since, by $(\mathrm{V}), \sum_{j=1}^{N} \Omega_{j}+\check{H}_{\mathrm{f}, \sigma}$ is also relatively bounded with respect to $\check{H}_{\sigma}^{V}, K$ is relatively compact with respect to $\check{H}_{\sigma}^{V}$. By the definition of $\hat{o}(\mathbb{1})$, there is a constant $C$ independent of $P$ and $R$ such that $\hat{o}(\mathbb{1}) \geq-o(\mathbb{1})\left(\check{H}_{\sigma}^{V}+C\right)$. Thus, we have the operator inequality

$$
(1+o(\mathbb{1})) \check{H}_{\sigma}^{V}-E_{\sigma}^{V}+o(\mathbb{1})-K \geq \sigma \chi_{R}^{2}+\left(\Sigma_{\sigma}^{V}-E_{\sigma}^{V}\right) \bar{\chi}_{R}^{2} \geq \min \left\{\sigma, \Sigma_{\sigma}^{V}-E_{\sigma}^{V}\right\} .
$$

Since $K$ does not change the essential spectrum of $\check{H}_{\sigma}^{V}$, for all $P$ and $R$ we have

$$
(1+o(\mathbb{1})) \inf \sigma_{\mathrm{ess}}\left(H_{\sigma}^{V}\right)-E_{\sigma}^{V}+o(\mathbb{1}) \geq \min \left\{\sigma, \Sigma_{\sigma}^{V}-E_{\sigma}^{V}\right\} .
$$

Hence, by (60),

$$
\inf \sigma_{\mathrm{ess}}\left(H_{\sigma}^{V}\right)-E_{\sigma}^{V} \geq \min \left\{\sigma, \Sigma_{\sigma}^{V}-E_{\sigma}^{V}\right\}>0
$$

Therefore $\sigma\left(\check{H}_{\sigma}^{V}\right) \cap\left[E_{\sigma}^{V}, E_{\sigma}^{V}+\min \left\{\sigma, \Sigma_{\sigma}^{V}-E_{\sigma}^{V}\right\}\right)$ is a purely discrete spectrum. In particular, $H_{\sigma}^{V}$ has a ground state.

\section{$\S 4.2$. Case $\sigma=0$}

Next we prove the existence of a ground state of $H^{V}$. For $\sigma>0$, let $\Phi_{\sigma} \in \mathscr{H}$ be a normalized ground state of $H_{\sigma}^{V}$. Let $\left\{\sigma_{n}\right\}$ be a sequence such that $\lim _{n \rightarrow \infty} \sigma_{n}=0$ and $\Phi_{\sigma_{n}}$ converges weakly to some vector $\Phi \in \mathscr{H}$. It is well known from [AH97] that if $\Phi \neq 0$ then $\Phi$ is a ground state of $H^{V}$. In the following we prove that a subsequence of $\left\{\Phi_{\sigma}\right\}_{\sigma}$ converges to some nonzero vector $\Phi$.

Lemma 4.5. We have the energy bound $\sup _{0<\sigma \ll 1}\left(\Phi_{\sigma}, H_{0} \Phi_{\sigma}\right)<\infty$. In addition, if $E^{V}<\Sigma^{V}$, then $\sup _{0<\sigma \ll 1}\left(\Phi_{\sigma}, N \Phi_{\sigma}\right)<\infty$.

Proof. The former statement follows from the definition of $\Phi_{\sigma}$, and the latter from [Ger00, Lemma IV2]. 
We denote by $B(\mathcal{K})$ the set of bounded operators on a Hilbert space $\mathcal{K}$. For each $k \in \mathbb{R}^{d}$, let

$$
v(k)=\sum_{j=1}^{N} \frac{\alpha_{j}}{\sqrt{2}} \hat{\lambda}_{j}(-k) e^{-i k x_{j}} .
$$

Then $v(k) \in B\left(L^{2}\left(\mathbb{R}_{X}^{d N}\right)\right)$. For each $k \in \mathbb{R}^{d}$, we set

$$
T(k)=\left(H^{V}-E^{V}+\omega(k)\right)^{-1}\left(v(k) \otimes \mathbb{1}_{\mathscr{F}}\right) .
$$

Then $T(k) \in B(\mathscr{H})$ for every $k \in \mathbb{R}^{d},(\Psi, T(k) \Phi)$ is measurable for all $\Phi, \Psi \in \mathscr{H}$, and $\int_{\mathbb{R}^{d}}\|T(k)\|_{B(\mathscr{H})}^{2} d k<\infty$. Hence $T(\cdot)$ can be regarded as a vector in the Banach space $L^{2}\left(\mathbb{R}^{d} ; B(\mathscr{H})\right)$. Since $\Phi_{\sigma} \in D\left(N^{1 / 2}\right), a(k) \Phi_{\sigma}$ is well defined for almost every $k \in \mathbb{R}^{d}$. Let $\theta_{s}, s \in \mathbb{R}^{d}$, be the shift on $L^{2}\left(\mathbb{R}^{d} ; B(\mathscr{H})\right)$, i.e., for $B \in L^{2}\left(\mathbb{R}^{d} ; B(\mathscr{H})\right)$,

$$
\left(\theta_{s} B\right)(k)=B(k-s), \quad \text { a.e. } k \in \mathbb{R}^{d} .
$$

Lemma 4.6. The map $\mathbb{R}^{d} \ni s \mapsto\left\|\theta_{s} T e^{-\delta|x|}\right\|_{L^{2}\left(\mathbb{R}^{d} ; B(\mathscr{H})\right)} \in \mathbb{R}$ is continuous.

Proof. Since $\theta_{s}$ is a translation, it is enough to show that $\left\|\theta_{s} T e^{\delta|x|}\right\|$ is continuous at $s=0$, i.e., $\left\|\theta_{s} T e^{-\delta|x|}-T e^{-\delta|x|}\right\|_{L^{2}\left(\mathbb{R}^{d} ; B(\mathscr{H})\right)}$ converges to 0 as $s \rightarrow 0$. We have

$$
\begin{aligned}
& \left.\left\|\theta_{s} T e^{-\delta|x|}-T e^{-\delta|x|}\right\|_{L^{2}\left(\mathbb{R}^{d} ; B(\mathscr{H})\right)}\right)\left\|T(k-s) e^{-\delta|x|}-T(k) e^{-\delta|x|}\right\|_{B(\mathscr{H})}^{2} \\
& \quad \leq\left(\int_{|k| \leq C_{1}}+\int_{|k| \geq C_{2}}+\int_{C_{1}<|k|<C_{2}}\right) \| T(k)
\end{aligned}
$$

for $0<C_{1}<C_{2}$. For $C_{1}<|k|<C_{2}$, we write

$$
\begin{aligned}
& T(k-s) e^{-\delta|x|}-T(k) e^{-\delta|x|} \\
& =\left(H^{V}-E^{V}+\omega(k)\right)^{-1}\left(\sum_{j=1}^{N} \Omega_{j}+\mathbb{1}\right)\left(\sum_{j=1}^{N} \Omega_{j}+\mathbb{1}\right)^{-1}(v(k-s)-v(k)) e^{-\delta|x|} \\
& +\left(H^{V}-E^{V}+\omega(k)\right)^{-1}\left(H^{V}-E^{V}+\omega(k-s)\right)^{-1} v(k-s)(\omega(k-s)-\omega(k)) e^{-\delta|x|} .
\end{aligned}
$$

Since for all $k$ with $C_{1}<|k|<C_{2}$,

$$
\sup _{C_{1} \leq|k|}\left\|\left(H^{V}-E^{V}+\omega(k)\right)^{-1}\left(\sum_{j=1}^{N} \Omega_{j}+\mathbb{1}\right)\right\|<\infty,
$$

we have

$$
\begin{aligned}
& \left\|T(k-s) e^{-\delta|x|}-T(k) e^{-\delta|x|}\right\|_{B(\mathscr{H})} \\
& \quad \leq C\left\|\left(\sum_{j=1}^{N} \Omega_{j}+\mathbb{1}\right)^{-1} e^{-\delta|x|}(v(k-s)-v(k))\right\|_{B(\mathscr{H})}+C\left\|e^{-\delta|x|} v(k-s)\right\|_{B(\mathscr{H})}
\end{aligned}
$$


for some constant $C>0$ depending on $C_{1}$ and $C_{2}$. Note that

$$
\left(\sum_{j=1}^{N} \Omega_{j}+\mathbb{1}\right)^{-1} e^{-\delta|x|}
$$

is compact. By Proposition 4.7 below, we have

$$
\lim _{s \rightarrow 0} \int_{C_{1}<|k|<C_{2}}\left\|\left(\sum_{j=1}^{N} \Omega_{j}+\mathbb{1}\right)^{-1} e^{-\delta|x|}(v(k-s)-v(k))\right\|_{B(\mathscr{H})}^{2} d k=0 .
$$

Next we see that

$$
\begin{aligned}
& \lim _{s \rightarrow 0} \int_{|k| \leq C_{1}}\left\|T(k-s) e^{-\delta|x|}-T(k) e^{-\delta|x|}\right\|_{B(\mathscr{H})}^{2} d k \\
& \quad \leq 2 \lim _{s \rightarrow 0} \int_{|k| \leq C_{1}}\left(\frac{|\hat{\lambda}(-k)|^{2}}{|\omega(k)|^{2}}+\frac{|\hat{\lambda}(-k+s)|^{2}}{|\omega(-k+s)|^{2}}\right) d k \leq 4 \int_{k \leq C_{1}} \frac{|\hat{\lambda}(-k)|^{2}}{\omega(k)^{2}} d k,
\end{aligned}
$$

and the right-hand side above converges to zero as $C_{1} \rightarrow 0$. Similarly,

$$
\lim _{C_{2} \rightarrow \infty} \lim _{s \rightarrow 0} \int_{|k| \geq C_{2}}\left\|T(k-s) e^{-\delta|x|}-T(k) e^{-\delta|x|}\right\|_{B(\mathscr{H})}^{2} d k=0 .
$$

Therefore, by combining (64)-(65), we complete the proof.

Proposition 4.7 ([Ger06, proof of Lemma 3.2]). Suppose $\mathbb{R}^{d} \ni k \mapsto m(k) \in$ $B\left(L^{2}\left(\mathbb{R}^{d N}\right)\right)$ is a weakly measurable map such that for all $0<C_{1}<C_{2}$,

$$
\int_{C_{1} \leq|k| \leq C_{2}}\|m(k)\|_{B\left(L^{2}\left(\mathbb{R}^{d N}\right)\right)}^{2} d k<\infty
$$

and let $R$ be a compact operator on $L^{2}\left(\mathbb{R}^{d N}\right)$. Then for all $0<C_{1}<C_{2}$,

$$
\lim _{s \rightarrow 0} \int_{C_{1}<|k|<C_{2}}\|R(m(k-s)-m(k))\|_{B\left(L^{2}\left(\mathbb{R}^{d N}\right)\right)}^{2} d k=0 .
$$

Lemma 4.8. Let $F \in C_{0}^{\infty}\left(\mathbb{R}^{d}\right)$ be a cutoff function with $0 \leq F \leq 1, F(s)=1$ for $|s| \leq 1 / 2, F(s)=0$ for $|s| \geq 1$. Let $F_{R}=F_{R}\left(-i \nabla_{k}\right)=F\left(-i \nabla_{k} / R\right)$. Then

$$
\lim _{R \rightarrow \infty} \sup _{0<\sigma \ll 1}\left(\Phi_{\sigma}, \mathrm{d} \Gamma\left(\mathbb{1}-F_{R}\right) \Phi_{\sigma}\right)=0 .
$$

Proof. It is shown in [Ger00, proof of Proposition IV.3] that

$$
\lim _{\sigma \rightarrow 0} \int_{\mathbb{R}^{d}}\left\|a(k) \Phi_{\sigma}-T(k) \Phi_{\sigma}\right\|_{\mathscr{H}}^{2} d k=0 .
$$

Then

$$
\left(\Phi_{\sigma}, \mathrm{d} \Gamma\left(\mathbb{1}-F_{R}\right) \Phi_{\sigma}\right)_{\mathscr{H}}=\int_{\mathbb{R}^{d}}\left(T(k) \Phi_{\sigma},\left(\mathbb{1}-F_{R}\right) T(k) \Phi_{\sigma}\right) \mathscr{H} d k+o\left(\sigma^{0}\right),
$$


where $o\left(\sigma^{0}\right)$ denotes a constant that converges to 0 as $\sigma \rightarrow 0$. By the CauchySchwarz inequality the right-hand side above has the upper bound

$$
\|T\|_{L^{2}\left(\mathbb{R}^{d} ; B(\mathscr{H})\right)} \cdot\left\|\left(\mathbb{1}-F_{R}\right) T(k) e^{-\delta|x|}\right\|_{L^{2}\left(\mathbb{R}_{k}^{d} ; B(\mathscr{H})\right)} \cdot\left\|e^{\delta|x|} \Phi_{\sigma}\right\|_{\mathscr{H}}+o\left(\sigma^{0}\right) .
$$

Note that $\sup _{0<\sigma \ll 1}\left\|e^{\delta|x|} \Phi_{\sigma}\right\|_{\mathscr{C}}<\infty$ for some $\delta>0$ by assumption. By the Fourier transformation, we have

$$
\begin{aligned}
\|\left(\mathbb{1}-F_{R}\right) & T(k) e^{-\delta|x|} \|_{L^{2}\left(\mathbb{R}^{d} ; B(\mathscr{H})\right)}^{2} \\
= & \int_{\mathbb{R}^{d}}\left\|(2 \pi)^{-d / 2} \int_{\mathbb{R}^{d}} d s \hat{F}(s)\left(\mathbb{1}-\theta_{-s / R}\right) T(k) e^{-\delta|x|}\right\|_{B(\mathscr{H})}^{2} d k \\
\leq & (2 \pi)^{-d} \int_{\mathbb{R}^{d}}|\hat{F}(s)|^{2} \cdot\left\|\left(\mathbb{1}-\theta_{-s / R}\right) T e^{-\delta|x|}\right\|_{L^{2}\left(\mathbb{R}^{d} ; B(\mathscr{H})\right)} d s .
\end{aligned}
$$

Notice that

$$
|\hat{F}(s)|^{2} \cdot\left\|\left(\mathbb{1}-\theta_{-s / R}\right) T e^{-\delta|x|}\right\|_{L^{2}\left(\mathbb{R}^{d} ; B(\mathscr{H})\right)} \leq|\hat{F}(s)|^{2} \cdot 2\left\|\hat{\lambda}_{j} / \omega\right\|,
$$

and the right-hand side above is integrable in $s$ and independent of $R$. Moreover, Lemma 4.6 implies that the last integrand in (68) converges to 0 as $R \rightarrow \infty$. Therefore, by the Lebesgue dominated convergence theorem, (68) converges to 0 as $R \rightarrow \infty$, and hence (66) holds.

Proposition 4.9. $H^{V}$ has a ground state.

Proof. The proof is parallel to that of [Ger00, Lemma IV.5]. From $\left(\mathbb{1}-\Gamma\left(F_{R}\right)\right)^{2} \leq$ $\mathrm{d} \Gamma\left(\mathbb{1}-F_{R}\right)$ and Lemma 4.8, we have

$$
\left\|\left(\mathbb{1}-\Gamma\left(F_{R}\right)\right) \Phi_{\sigma}\right\| \leq o\left(R^{0}\right)+o\left(\sigma^{0}\right) .
$$

Let $\left\{\sigma_{n}\right\}_{n}$ be a subsequence such that $\lim _{n \rightarrow \infty} \sigma_{n}=0$ and $\Phi=\mathrm{w}-\lim _{n \rightarrow \infty} \Phi_{\sigma_{n}}$. By Lemmas 4.5 and 3.8, and (69), for every $\varepsilon>0$ there exist $R_{0}, \lambda_{0}, n_{0}>0$ such that for all $R>R_{0}, \lambda_{0}>\lambda$ and $n \geq n_{0}$,

$$
\begin{aligned}
& \left\|\left(\mathbb{1}-\chi\left(H_{0} \leq \lambda\right)\right) \Phi_{\sigma_{n}}\right\|<\varepsilon, \quad\left\|(\mathbb{1}-\chi(N \leq \lambda)) \Phi_{\sigma_{n}}\right\|<\varepsilon, \\
& \left\|(\mathbb{1}-\chi(|X| \leq \lambda)) \Phi_{\sigma_{n}}\right\|<\varepsilon, \quad\left\|\left(\mathbb{1}-\Gamma\left(F_{R}\right)\right) \Phi_{\sigma_{n}}\right\|<\varepsilon,
\end{aligned}
$$

where $\chi(s \leq \lambda)$ denotes the characteristic function of $\{s \in \mathbb{R} \mid s \leq \lambda\}$. Note that $K=\chi\left(H_{0} \leq \lambda\right) \chi(N \leq \lambda) \chi(|X| \leq \lambda) \Gamma\left(F_{R}\right)$ is a compact operator. For all large $R, \lambda>0$, we have

$$
\begin{aligned}
\|\Phi\| & \geq\|K \Phi\|-\|(\mathbb{1}-K) \Phi\| \geq \lim _{n \rightarrow \infty}\left\|K \Phi_{\sigma_{n}}\right\|-\|(\mathbb{1}-K) \Phi\| \\
& \geq \liminf _{n \rightarrow \infty}\left(\left\|\Phi_{\sigma_{n}}\right\|-\left\|(\mathbb{1}-K) \Phi_{\sigma_{n}}\right\|\right)-\|(\mathbb{1}-K) \Phi\| \geq 1-4 \varepsilon-\|(\mathbb{1}-K) \Phi\| .
\end{aligned}
$$


Clearly $\mathbb{1}-K$ strongly converges to 0 when $R$ and $\lambda$ go to infinity. Since $\varepsilon>0$ is arbitrary, we have $\|\Phi\|=1$. Therefore $H^{V}$ has a normalized ground state $\Phi$.

\section{§5. Essential spectrum}

We state without proofs some general lemmas given in [HS08].

Lemma 5.1. Let $K_{\epsilon}, \epsilon>0$, and $K$ be self-adjoint operators on a Hilbert space $\mathcal{K}$, and $\sigma_{\mathrm{ess}}\left(K_{\epsilon}\right)=\left[\xi_{\epsilon}, \infty\right)$. Suppose that $\lim _{\epsilon \rightarrow 0} K_{\epsilon}=K$ in the uniform resolvent sense, and $\lim _{\epsilon \rightarrow 0} \xi_{\epsilon}=\xi$. Then $\sigma_{\mathrm{ess}}(K)=[\xi, \infty)$. In particular, $\lim _{\epsilon \rightarrow 0} \inf \sigma_{\mathrm{ess}}\left(K_{\epsilon}\right)$ $=\inf \sigma_{\text {ess }}(K)$.

Lemma 5.2. Let $\Delta$ be the d-dimensional Laplacian. Assume that $V(-\Delta+1)^{-1 / 2}$ is a compact operator. Then there exists a sequence $\left\{V^{\epsilon}\right\}_{\epsilon>0}$ such that $V^{\epsilon} \in$ $C_{0}^{\infty}\left(\mathbb{R}^{d}\right)$ and $\lim _{\epsilon \rightarrow 0} V^{\epsilon}(-\Delta+1)^{-1 / 2}=V(-\Delta+1)^{-1 / 2}$ uniformly.

Set

$$
k_{0}(\beta)=-\sum_{j \in \beta} \sqrt{-\Delta_{j}}+\sum_{i, j \in \beta} V_{i j}, \quad k_{V}(\beta)=h_{0}(\beta)+\sum_{j \in \beta} V_{j}
$$

with $V_{i}, V_{i j} \in L_{\text {loc }}^{2}\left(\mathbb{R}^{d}\right)$ such that $V_{i}(-\Delta+1)^{-1 / 2}$ and $V_{i j}(-\Delta+1)^{-1 / 2}$ are compact operators. We define $K=k_{V}\left(C_{N}\right)$. Let

$$
\Xi_{V}=\min _{\beta \subsetneq C_{N}}\left\{\inf \sigma\left(k_{0}(\beta)\right)+\inf \sigma\left(k_{V}(\beta)\right)\right\}
$$

be the lowest two-cluster threshold of $K$.

Lemma 5.3. There exist sequences $\left\{V_{i}^{\epsilon}\right\}_{\epsilon},\left\{V_{i j}^{\epsilon}\right\}_{\epsilon} \subset C_{0}^{\infty}\left(\mathbb{R}^{d}\right), i, j=1, \ldots, N$, such that

$$
\text { (1) } \lim _{\epsilon \rightarrow 0} \Xi_{V}(\epsilon)=\Xi_{V}, \quad \text { (2) } \lim _{\epsilon \rightarrow 0} \inf \sigma_{\text {ess }}(K(\epsilon))=\inf \sigma_{\text {ess }}(K),
$$

where $\Xi_{V}(\epsilon)$ (resp. $\left.K(\epsilon)\right)$ is $\Xi_{V}$ (resp. $K$ ) with $V_{i}$ and $V_{i j}$ replaced by $V_{i}^{\epsilon}$ and $V_{i j}^{\epsilon}$, respectively.

\section{$\S 6$. Functional integration and energy comparison inequality}

In this Appendix we shall show Lemma 3.2 and Proposition 3.4 by functional integration. To do so, we take a Schrödinger representation instead of the Fock representation. We quickly review the former.

Let $\mathscr{Q}=\mathscr{S}_{\mathbb{R}}^{\prime}\left(\mathbb{R}^{d}\right)$ be the set of real-valued Schwartz distributions on $\mathbb{R}^{d}$. The boson Fock space $\mathscr{F}$ can be identified with $L^{2}(\mathscr{Q}, \mu)$ with some Gaussian measure $\mu$ 
such that

$$
\mathbb{E}_{\mu}[\phi(f)]=0, \quad \mathbb{E}_{\mu}[\phi(f) \phi(g)]=\frac{1}{2}(f, g)
$$

for $f, g \in L_{\mathbb{R}}^{2}\left(\mathbb{R}^{d}\right)$. Then the scalar field operator in $\mathscr{F}$ is unitarily equivalent to the Gaussian random variable $\phi(f)$ in $L^{2}(\mathscr{Q})$ :

$$
\phi(f) \sim \frac{1}{\sqrt{2}} \int\left(a^{*}(k) \hat{f}(-k)+a(k) \hat{f}(k)\right) d k
$$

for $f \in L_{\mathbb{R}}^{2}\left(\mathbb{R}^{d}\right)$. Moreover $H_{\mathrm{f}}$ can be unitarily transformed into a self-adjoint operator in $L^{2}(\mathscr{Q})$. We denote it by the same notation, $H_{\mathrm{f}}$.

Furthermore we need the Euclidean quantum field to construct the functional integral representation of the one-parameter semigroup generated by the Nelson Hamiltonian $H^{V}$. Set $\mathscr{Q}_{E}=\mathscr{S}_{\mathbb{R}}^{\prime}\left(\mathbb{R}^{d+1}\right)$. Thus $L^{2}\left(\mathscr{Q}_{E}, \mu_{E}\right)$ is the $L^{2}$ space endowed with a Gaussian measure such that

$$
\mathbb{E}_{\mu_{E}}\left[\phi_{E}(F)\right]=0, \quad \mathbb{E}_{\mu_{E}}\left[\phi_{E}(F) \phi_{E}(G)\right]=\frac{1}{2}(F, G)_{L^{2}\left(\mathbb{R}^{d+1}\right)}
$$

Let $j_{t}: L_{\mathbb{R}}^{2}\left(\mathbb{R}^{d}\right) \rightarrow L_{\mathbb{R}}^{2}\left(\mathbb{R}^{d+1}\right)$ be a family of isometries connecting $L^{2}(\mathscr{Q})$ and $L^{2}\left(\mathscr{Q}_{E}\right)$ which satisfies

$$
j_{s}^{*} j_{t}=e^{-|t-s| \omega(-i \nabla)}
$$

for all $s, t \in \mathbb{R}$. Let $J_{s}=\Gamma\left(j_{s}\right)$ be the second quantization of $j_{s}$. Then $J_{s}: L^{2}(\mathscr{Q}) \rightarrow$ $L^{2}\left(\mathscr{Q}_{E}\right)$ is also a family of isometries such that $J_{s}^{*} J_{t}=e^{-|t-s| H_{\mathrm{f}}}$ for all $s, t \in \mathbb{R}$. We identify $\mathscr{H}$ with the set of $L^{2}(\mathscr{Q})$-valued $L^{2}$ functions on $\mathbb{R}^{d N}, \int_{\mathbb{R}^{d N}}^{\oplus} L^{2}(\mathscr{Q}) d X$, and $H^{V}$ can be expressed as

$$
H_{\mathrm{p}} \otimes \mathbb{1}+\kappa^{2} \mathbb{1} \otimes H_{\mathrm{f}}+\kappa \alpha \sum_{j=1}^{N} \int_{\mathbb{R}^{d N}}^{\oplus} \phi\left(\lambda\left(\cdot-x_{j}\right)\right) d X
$$

in the Schrödinger representation.

Next we prepare a probabilistic description of the self-adjoint operator $H_{\mathrm{p}}$. Let $\left(X_{t}\right)_{t \geq 0}=\left(X_{t}^{1}, \ldots, X_{t}^{N}\right)_{t \geq 0}$ be the $\mathbb{R}^{d N}$-valued Lévy processes on a probability space $\left(\mathcal{D}, B, \mathrm{P}^{x}\right)$ starting from $x=0$ with characteristic function (34). Set $W\left(x_{1}, \ldots, x_{N}\right)=\sum_{j=1}^{N} V\left(x_{j}\right)$. Then we have the Feynman-Kac formula

$$
\left(f, e^{-H_{\mathrm{p}}} g\right)=\int_{\mathbb{R}^{d N}} \mathbb{E}_{\mathrm{P}}^{x}\left[\bar{f}\left(X_{0}\right) g\left(X_{t}\right) e^{-\int_{0}^{t} W\left(X_{s}\right) d s}\right] .
$$

The functional integral representation of $e^{-t H^{V}}$ can be obtained in the same way as the standard Nelson model. The only difference is the process associated with the kinetic term. Instead of the Brownian motion the Lévy process $\left(X_{t}^{j}\right)_{t \geq 0}$ is taken for $e^{-t H^{V}}$. The Feynman-Kac type formula for $e^{-t H^{V}}$ is then given by 
$\left(F, e^{-t H^{V}} G\right)_{\mathscr{H}}=$

$\int_{\mathbb{R}^{d N}} d x \mathbb{E}_{\mathrm{P}}^{x}\left[e^{-\int_{0}^{t} W\left(X_{s}\right) d s}\left(J_{0} F\left(X_{0}\right), e^{-\kappa \phi_{E}\left(\sum_{j=1}^{N} \int_{0}^{t} j_{\kappa^{2} s} \lambda_{j}\left(\cdot-X_{s}\right) d s\right)} J_{\kappa^{2} t} G\left(X_{t}\right)\right)_{L^{2}\left(\mathscr{Q}_{E}\right)}\right]$.

Next we also consider the Feynman-Kac formula for $\exp \left(-t e^{-i T} H^{V} e^{i T}\right)$. It is given by the composition of $d N$-dimensional Brownian motion $\left(B_{t}^{1}, \ldots, B_{t}^{N}\right)_{t \geq 0}$ on a probability space $\left(\mathcal{C}, \mathcal{B}, \mathrm{W}^{x}\right)$ and $N$ independent subordinators $\left(T_{t}^{j}\right)_{t \geq 0}, j=$ $1, \ldots, N$, on $\left(\Omega_{\mu}, \mathcal{B}_{\mu}, \mu\right)$ such that $B_{T_{t}^{j}}^{j}$ has the same distribution as $X_{t}^{j}$. Set $B_{T_{t}}=$ $\left(B_{T_{t}^{j}}^{j}\right)_{t \geq 0, j=1, \ldots, N}$. We have

Proposition 6.1. Let $F, G \in \mathscr{H}$. Then

$\left(F, e^{-t e^{-i T} H^{V} e^{i T}} G\right)=e^{t E_{\text {diag }}} \int_{\mathbb{R}^{d N}} d x \mathbb{E}_{\mathrm{W} \times \mu}^{x, 0}\left[e^{-\int_{0}^{t}\left(W+V_{\text {eff }}\right)\left(B_{T_{s}}\right) d s}\right.$

$$
\left.\times\left(J_{0} F\left(B_{T_{0}}\right), e^{-i \kappa^{-1} \phi_{E}\left(K_{t}\right)} J_{\kappa^{2} t} G\left(B_{T_{t}}\right)\right)_{L^{2}\left(\mathscr{Q}_{E}\right)}\right] .
$$

Here $K_{t}=\sum_{j=1}^{N} \int_{0}^{T_{t}^{j}} j_{\left(T^{j-1}\right)_{\kappa^{2}}} \lambda_{j}\left(\cdot-B_{s}^{j}\right) \circ d B_{s}^{j}$ denotes the $L^{2}\left(\mathbb{R}^{d+1}\right)$-valued Stratonovich integral and $j_{\left(T^{j-1}\right)_{t}}$ are some isometries defined by $\left(T_{t}^{j}\right)_{t \geq 0}$.

Proof. See [Hir14, Theorem 3.15].

By using Proposition 6.1 we can compute the scaling limit of $e^{-i T} H^{V} e^{i T}$ as $\kappa \rightarrow \infty$. Note that $\left(J_{0} \Phi, J_{\kappa^{2} t} \Psi\right) \rightarrow\left(\Phi, P_{\Omega} \Phi\right)$ as $\kappa \rightarrow \infty$ for $t \neq 0$. Then by the functional integral representation (Proposition 6.1) we immediately see that

$$
\lim _{\kappa \rightarrow \infty}\left(F, e^{-t e^{-i T} H^{V} e^{i T}} G\right)=\left(F, e^{-t\left(h_{\mathrm{eff}}^{V}-E_{\mathrm{diag}}\right)} \otimes P_{\Omega} G\right) .
$$

Since $h_{\text {eff }}^{V}$ has a ground state, this suggests that $H^{V}$ also has a ground state for sufficiently large $\kappa$. This has been indeed proved in Section 3 .

By functional integral representation we have an energy comparison bound.

Proposition 6.2. We have $\inf \sigma\left(H^{V}\right) \leq \inf \sigma\left(h_{\text {eff }}^{V}\right)+E_{\text {diag }}$.

Proof. By Proposition 6.1,

$$
\left.\left|\left(F, e^{-t e^{-i T} H^{V} e^{i T}} G\right)\right| \leq e^{t E_{\text {diag }}}\left(|F|, e^{-t\left(h_{\text {eff }}^{V}+H_{\mathrm{f}}\right.}\right)|G|\right) .
$$

Hence the proposition follows.

In the same way as with Proposition 6.2 but for $H^{V}$ replaced by $H^{V}(\beta)$ or $H^{0}(\beta)$ we obtain

Proposition 6.3 (Lemma 3.2). We have

$$
\inf \sigma\left(H^{\#}(\beta)\right) \leq \inf \sigma\left(h_{\text {eff }}^{\#}(\beta)\right)+\sum_{j \in \beta} \frac{\alpha^{2}}{2}\left\|\hat{\lambda}_{j} / \sqrt{\omega}\right\|^{2}, \quad \#=0, V .
$$


Next we prove Proposition 3.4. We can construct the functional integral representation of $e^{-t H_{\sigma}^{V}}$ in much the same way as that of $e^{-t H^{V}}$. The only difference is to replace $\hat{\lambda}_{j}$ with $\hat{\lambda}_{j}\lceil\omega(k)>\sigma$.

Proposition 6.4. Proposition 3.4 holds.

Proof. Notice that $\Phi_{\sigma}=e^{-t\left(e^{-i T} H_{\sigma}^{V} e^{i T}-E_{\sigma}^{V}\right)} \Phi_{\sigma}$. Then by Proposition 6.1,

$$
\Phi_{\sigma}(x)=e^{t\left(E_{\sigma}^{V}+E_{\mathrm{diag}}\right)} \mathbb{E}_{\mathrm{W} \times \mu}^{x, 0}\left[e^{-\int_{0}^{t} W_{\mathrm{eff}}\left(B_{T_{s}}\right) d s} J_{0}^{*} e^{-i \kappa^{-1} \phi_{E}\left(K_{t}\right)} J_{\kappa^{2} t} \Phi_{\sigma}\left(B_{T_{t}}\right)\right] .
$$

Thus it is straightforward to see by the Schwarz inequality that

$$
\left\|\Phi_{\sigma}(x)\right\|_{\mathscr{F}} \leq e^{t\left(E_{\sigma}^{V}+E_{\mathrm{diag}}\right)}\left(\mathbb{E}_{\mathrm{W} \times \mu}^{x, 0}\left[e^{-2 \int_{0}^{t} W_{\mathrm{eff}}\left(B_{T_{s}}\right) d s}\right]\right)^{1 / 2}\left\|\Phi_{\sigma}\right\|_{\mathscr{H}}
$$

Note that $\lim _{\sigma \rightarrow 0} E_{\sigma}^{V}=E^{V}$. Then the proposition follows, since $B_{T_{t}}$ has the same distribution as $X_{t}$.

\section{$\S 7$. The bound $E(0) \leq E(P)$ and continuity of $E(\cdot)$}

We next consider a fiber decomposition of the translation invariant relativistic Schrödinger operator $H_{\mathrm{p}}=\sum_{j=1}^{N} \Omega_{j}+V_{\text {eff }}$ in $L^{2}\left(\mathbb{R}^{d N}\right)$.

For notational convenience and generalizations, we consider the Schrödinger operator of the form $H_{\mathrm{p}}=\sum_{j=0}^{N} \Omega_{j}+v$ in $L^{2}\left(\mathbb{R}^{d(N+1)}\right)$, where

$$
v=\sum_{j=0}^{N} v_{i j}\left(x_{i}-x_{j}\right)
$$

and we assume that $v$ is relativistic of Kato class. Let $X_{t}=\left(X_{t}^{j}\right)_{t \geq 0}, j=0, \ldots, N$, be $N+1$ independent Lévy processes with $\mathbb{E}_{\mathrm{P}}^{x}\left[e^{i u \cdot X_{t}^{j}}\right]=e^{-t \Omega_{j}(u)}$, and set $\mathbf{X}_{t}=$ $\left(X_{t}^{j}\right)_{t \geq 0, j=1, \ldots, N}$. Let $P_{\text {tot }}=\sum_{j=0}^{N} p_{j}$ be the total momentum. Then $H_{\mathrm{p}}$ commutes with $P_{\text {tot }}$, and so $H_{\mathrm{p}} \cong \int_{\mathbb{R}^{d}}^{\oplus} k(P) d P$, where $k(P)$ is a self-adjoint operator on $L^{2}\left(\mathbb{R}^{d N}\right)$. Let $E(P)=\inf \sigma(k(P))$.

Theorem 7.1. (1) $E(0) \leq E(P)$ for all $P \in \mathbb{R}^{d}$.

(2) $\mathbb{R}^{d} \ni P \mapsto E(P) \in \mathbb{R}$ is continuous.

We shall prove this theorem by making use of a path integral representation. Set $x=\left(x_{0}, \mathbf{x}\right) \in \mathbb{R}^{d} \times \mathbb{R}^{d N}$. Let $U=F e^{i x_{0} \cdot \sum_{j=1}^{N} p_{j}}: L^{2}\left(\mathbb{R}^{d(N+1)}\right) \rightarrow L^{2}\left(\mathbb{R}^{d(N+1)}\right)$ be a unitary operator, where $F$ denotes the Fourier transformation with respect to $x_{0}$, i.e., $F f(k, \mathbf{x})=(2 \pi)^{-d / 2} \int f\left(x_{0}, \mathbf{x}\right) e^{-i k \cdot x_{0}} d x_{0}$. We have

$$
(U f)(k, \mathbf{x})=(2 \pi)^{-d / 2} \int_{\mathbb{R}^{d}} e^{-i k \cdot x_{0}} f\left(x_{0}, x_{1}+x_{0}, \ldots, x_{N}+x_{0}\right) d x_{0} .
$$


Thus we can directly see that $\left(U P_{\text {tot }} U^{-1} f\right)(k, \mathbf{x})=k f(k, \mathbf{x})$. Hence $U$ diagonalizes $P_{\text {tot }}$, and thus $U H_{\mathrm{p}} U^{-1}=\int_{\mathbb{R}^{d}} k(P) d P$. We have

$$
\left(f, e^{-t H_{\mathrm{p}}} g\right)_{L^{2}\left(\mathbb{R}^{d(N+1)}\right)}=\int_{\mathbb{R}^{d(N+1)}} d x \mathbb{E}_{\mathrm{P}}^{\left(x_{0}, \mathbf{x}\right)}\left[\overline{f\left(X_{0}\right)} g\left(X_{t}\right) e^{-\int_{0}^{t} v\left(X_{s}\right) d s}\right] .
$$

We construct the Feynman-Kac formula for $\left(f, e^{-t k(P)} g\right)_{L^{2}\left(\mathbb{R}^{d N}\right)}$. Let $v=0$. Then

$$
k(P)=\Omega_{0}\left(P-\sum_{j=1}^{N} p_{j}\right)+\sum_{j=1}^{N} \Omega_{j}\left(p_{j}\right) .
$$

Since $\mathbb{E}_{\mathrm{P}}^{(0, \mathbf{x})}\left[e^{i X_{t}^{0}\left(P-\sum_{j=1}^{N} p_{j}\right)}\right]=e^{-t \Omega_{0}\left(P-\sum_{j=1}^{N} p_{j}\right)}$, we intuitively see that

$$
\left(f, e^{-t k(P)} g\right)_{L^{2}\left(\mathbb{R}^{d N}\right)}=\int_{\mathbb{R}^{d N}} d \mathbf{x} \mathbb{E}_{\mathrm{P}}^{(0, \mathbf{x})}\left[\overline{f\left(\mathbf{X}_{0}\right)} e^{i X_{t}^{0} \cdot\left(P-\sum_{j=1}^{N} p_{j}\right)} g\left(\mathbf{X}_{t}\right)\right] .
$$

Note that $e^{-i X_{t}^{0} \cdot \sum_{j=1}^{N} p_{j}}$ denotes a translation:

$$
\left(e^{-i X_{t}^{0} \cdot \sum_{j=1}^{N} p_{j}} g\right)\left(\mathbf{X}_{t}\right)=g\left(X_{t}^{1}-X_{t}^{0}, \ldots, X_{t}^{N}-X_{t}^{0}\right) .
$$

The next proposition gives the Feynman-Kac formula with potential.

Proposition 7.2. Let $F, G \in L^{2}\left(\mathbb{R}^{d N}\right)$ and $P \in \mathbb{R}^{d}$. Then

$$
\begin{aligned}
& \left(F, e^{-t k(P)} G\right)_{L^{2}\left(\mathbb{R}^{d N}\right)} \\
& \quad=\int_{\mathbb{R}^{d N}} d \mathbf{x} \mathbb{E}_{\mathrm{P}}^{(0, \mathbf{x})}\left[\overline{F\left(\mathbf{X}_{0}\right)} e^{-\int_{0}^{t} v\left(X_{s}\right) d s} e^{i X_{t}^{0} \cdot\left(P-\sum_{j=1}^{N} p_{j}\right)} G\left(\mathbf{X}_{t}\right)\right]
\end{aligned}
$$

Proof. Let $\xi \in \mathbb{R}^{d}$. First we see that

$$
\left(f, e^{-t H_{\mathrm{p}}} e^{i \xi \cdot P_{\mathrm{tot}}} g\right)_{L^{2}\left(\mathbb{R}^{d(N+1)}\right)}=\int_{\mathbb{R}^{d}} d P e^{i \xi \cdot P}\left(f(P), e^{-t k(P)} g(P)\right)_{L^{2}\left(\mathbb{R}^{d N}\right)},
$$

where

$$
f(P)=(U f)(P, \mathbf{x})=(2 \pi)^{-d / 2} \int_{\mathbb{R}^{d}} e^{-i P \cdot X} f\left(X, x_{1}+X, \ldots, x_{N}+X\right) d X,
$$

and $g(P)$ is given similarly. Now we set $f=f_{s}=p_{s} \otimes F$ and $g=g_{r}=p_{r} \otimes G$, where $F, G \in \mathscr{S}\left(\mathbb{R}^{3 N}\right)$ and $p_{s}(X)=(2 \pi s)^{-d} \exp \left(-|X|^{2} /(2 s)\right)$ is the heat kernel. Note that $f_{s} \rightarrow \delta\left(x_{0}\right) \otimes F$ as $s \downarrow 0$. We have

$$
\begin{aligned}
\lim _{s \downarrow 0} \int_{\mathbb{R}^{d}} d P e^{i \xi \cdot P}\left(f_{s}(P), e^{-t k(P)} g_{r}(P)\right)_{L^{2}\left(\mathbb{R}^{d N}\right)} & \\
& =(2 \pi)^{-d / 2} \int_{\mathbb{R}^{d}} d P e^{i \xi \cdot P}\left(F, e^{-t k(P)} g_{r}(P)\right)_{L^{2}\left(\mathbb{R}^{d N}\right)} .
\end{aligned}
$$


The right-hand side above is the inverse Fourier transform of the function $h: P \mapsto$ $\left(F, e^{-t k(P)} g_{r}(P)\right)_{L^{2}\left(\mathbb{R}^{d N}\right)}$ and

$$
\lim _{r \downarrow 0} h(P)=\left(F, e^{-k(P)} G\right)_{L^{2}\left(\mathbb{R}^{d N}\right)}(2 \pi)^{-d / 2} .
$$

On the other hand, the left-hand side of (76) can be represented by the FeynmanKac formula:

$$
\begin{aligned}
& \left(f_{s}, e^{-t H_{\mathrm{p}}} e^{i \xi \cdot P_{\text {tot }}} g_{r}\right) \\
& \quad=\int_{\mathbb{R}^{d(N+1)}} d x \mathbb{E}_{\mathrm{P}}^{\left(x_{0}, \mathbf{x}\right)}\left[\overline{f_{s}\left(X_{0}\right)} e^{-\int_{0}^{t} v\left(X_{s}\right) d s} g_{r}\left(X_{t}^{0}+\xi, \ldots, X_{t}^{N}+\xi\right)\right] .
\end{aligned}
$$

Taking $s \downarrow 0$, we have

$$
\begin{aligned}
& \int_{\mathbb{R}^{d(N+1)}} d x \mathbb{E}_{\mathrm{P}}^{\left(x_{0}, \mathbf{x}\right)}\left[\overline{f_{s}\left(X_{0}\right)} e^{-\int_{0}^{t} v\left(X_{s}\right) d s} g_{r}\left(X_{t}^{0}+\xi, \ldots, X_{t}^{N}+\xi\right)\right] \\
& \rightarrow \mathbb{E}_{\mathrm{P}}^{(0, \mathbf{0})}\left[\int_{\mathbb{R}^{d N}} d \mathbf{x} \overline{F(\mathbf{x})} e^{-\int_{0}^{t} v\left(X_{s}+(0, \mathbf{x})\right) d s} g_{r}\left(X_{t}^{0}+\xi, X_{t}^{1}+\xi+x_{1}, \ldots, X_{t}^{N}+\xi+x_{N}\right)\right] .
\end{aligned}
$$

The right-hand side is a function of $\xi$. Its Fourier transform with respect to $\xi$ is

$$
\begin{aligned}
\mathbb{E}_{\mathrm{P}}^{(0, \mathbf{0})}\left[\int_{\mathbb{R}^{d N}} d \mathbf{x} \overline{F(\mathbf{x})} e^{-\int_{0}^{t} v\left(X_{s}+(0, \mathbf{x})\right) d s}\right. \\
\left.\quad \times(2 \pi)^{-d / 2} \int_{\mathbb{R}^{d}} d \xi e^{-i \xi \cdot P} g_{r}\left(X_{t}^{0}+\xi, X_{t}^{1}+\xi+x_{1}, \ldots, X_{t}^{N}+\xi+x_{N}\right)\right] .
\end{aligned}
$$

Take $r \downarrow 0$. We have

$$
\begin{aligned}
\mathbb{E}_{\mathrm{P}}^{(0, \mathbf{0})}\left[\int_{\mathbb{R}^{d N}} d \mathbf{x} \overline{F(\mathbf{x})} e^{-\int_{0}^{t} v\left(X_{s}+(0, \mathbf{x})\right) d s} e^{i X_{t}^{0} \cdot P}\right. & \\
& \left.\times G\left(X_{t}^{1}-X_{t}^{0}+x_{1}, \ldots, X_{t}^{N}-X_{t}^{0}+x_{N}\right)\right] \\
= & \mathbb{E}_{\mathrm{P}}^{(0, \mathbf{x})}\left[\int_{\mathbb{R}^{d N}} d \mathbf{x} \overline{F\left(X_{0}\right)} e^{-\int_{0}^{t} v\left(X_{s}\right) d s} e^{i X_{t}^{0}\left(P-\sum_{j=1}^{N} p_{j}\right)} G\left(\mathbf{X}_{t}\right)\right] .
\end{aligned}
$$

Comparing (77) with the right-hand side above, we deduce the theorem for $F, G$ in $\mathscr{S}$. By a limiting argument the theorem is valid for all $f, g \in L^{2}\left(\mathbb{R}^{d N}\right)$.

Proof of Theorem 7.1. By Proposition 7.2 we have

$$
\left|\left(f, e^{-t k(P)} g\right)\right| \leq \int_{\mathbb{R}^{d N}} d x \mathbb{E}_{\mathrm{P}}^{(0, \mathbf{x})}\left[\left|f\left(\mathbf{X}_{0}\right)\right| e^{-\int_{0}^{t} v\left(X_{s}\right) d s}\left|e^{-i X_{t}^{0} \cdot \sum_{j=1}^{N} p_{j}} g\left(\mathbf{X}_{t}\right)\right|\right] .
$$

Since $e^{-i X_{t}^{0} \cdot \sum_{j=1}^{N} p_{j}}$ is a shift operator,

$$
\left|e^{-i X_{t}^{0} \cdot \sum_{j=1}^{N} p_{j}} g\left(\mathbf{X}_{t}\right)\right| \leq e^{-i X_{t}^{0} \cdot \sum_{j=1}^{N} p_{j}}\left|g\left(\mathbf{X}_{t}\right)\right| .
$$

Hence $\left|\left(f, e^{-t k(P)} g\right)\right| \leq\left(|f|, e^{-t k(0)}|g|\right)$, which yields (1). 
Next we show (2). By the Feynman-Kac formula it is immediate that

$$
\begin{aligned}
& \left(F,\left(e^{-t k(P)}-e^{-t k(Q)}\right) G\right) \\
& \quad=\int_{\mathbb{R}^{d N}} d \mathbf{x} \mathbb{E}_{\mathrm{P}}^{(0, \mathbf{x})}\left[\overline{F\left(\mathbf{X}_{0}\right)} e^{-\int_{0}^{t} v\left(X_{s}\right) d s} e^{-i X_{t}^{0} \cdot \sum_{j=1}^{N} p_{j}}\left(i \int_{X_{t}^{0} \cdot Q}^{X_{t}^{0} \cdot P} e^{i \theta} d \theta\right) G\left(\mathbf{X}_{t}\right)\right] .
\end{aligned}
$$

Therefore

$$
\frac{\left|\left(F,\left(e^{-t k(P)}-e^{-t k(Q)}\right) G\right)\right|}{\|F\|\|G\|} \leq|P-Q| \sup _{\mathbf{x} \in \mathbb{R}^{d N}}\left(\mathbb{E}_{\mathrm{P}}^{(0, \mathbf{x})}\left[\left|X_{t}^{0}\right|^{2} e^{-2 \int_{0}^{t} v\left(X_{s}\right) d s}\right]\right)^{1 / 2} .
$$

Since $v$ is relativistic of Kato class,

$$
\begin{aligned}
\sup _{\mathbf{x} \in \mathbb{R}^{d N}} \mathbb{E}_{\mathrm{P}}^{(0, \mathbf{x})}\left[\left|X_{t}^{0}\right|^{2} e^{-2 \int_{0}^{t} v\left(X_{s}\right) d s}\right] \\
\leq \sup _{\mathbf{x} \in \mathbb{R}^{d N}} \mathbb{E}_{\mathrm{P}}^{(0, \mathbf{x})}\left[\left|X_{t}^{0}\right|^{4}\right]^{1 / 2} \sup _{\mathbf{x} \in \mathbb{R}^{d N}}\left(\mathbb{E}_{\mathrm{P}}^{(0, \mathbf{x})}\left[e^{-4 \int_{0}^{t} v\left(X_{s}\right) d s}\right]\right)^{1 / 2}<\infty .
\end{aligned}
$$

Thus $e^{-t k(P)}$ uniformly converges to $e^{-t k(Q)}$ as $|P-Q| \rightarrow 0$, and (2) follows.

\section{Acknowledgments}

FH acknowledges support of Grant-in-Aid for Science Research (B) 20340032 from JSPS, Grant-in-Aid for Challenging Exploratory Research 22654018 and Grant-in-Aid for Challenging Exploratory Research 15K13445 from JSPS. He thanks for the hospitality of ICMS Edinburgh, where this work was partially done. IS acknowledges support of Grant-in-Aid for Young Scientists (B) 22740087 from JSPS. He thanks for the hospitality of Orsay University, where this work was partially done. IS's work is also supported by the program for dissemination of tenure-track system funded by the Ministry of Education and Science, Japan.

\section{References}

[AH97] A. Arai and M. Hirokawa, On the existence and uniqueness of ground states of a generalized spin-boson model, J. Funct. Anal. 151 (1997), 455-503. Zbl 0898.47048 MR 1491549

[AK03] A. Arai and H. Kawano, Enhanced binding in a general class of quantum field models, Rev. Math. Phys. 15 (2003), 387-423. Zbl 1081.81541 MR 1989538

[BFS98] V. Bach and J. Fröhlich and I. M. Sigal, Quantum electrodynamics of confined nonrelativistic particles, Adv. Math. 137 (1998), 299-395. Zbl 0923.47040 MR 1639713

[BLV05] J. M. Barbaroux, H. Linde and S. Vugalter, Qualitative estimates on the enhanced binding for the Pauli-Fierz operator, J. Math. Phys. 46 (2005), 122103, 11 pp. Zbl 1111.81337 MR 2194014

[BV04] R. D. Benguria and S. A. Vugalter, Binding threshold for the Pauli-Fierz operator, Lett. Math. Phys. 70 (2004), 249-257. Zbl 1078.81016 MR 2128953 
[CMS90] R. Carmona, W. C. Masters and B. Simon, Relativistic Schrödinger operators: asymptotic behavior of the eigenvalues, J. Funct. Anal. 91 (1990), 117-142. Zbl 0716.35006 MR 1054115

[CEH03] I. Catto, P. Exner and C. Hainzl, Enhanced binding revisited for a spinless particle in non-relativistic QED, J. Math. Phys. 45 (2004), 4174-4185. Zbl 1064.81110 MR 2098124

[CVV03] T. Chen, V. Vougalter and S. A. Vugalter, The increase of binding energy and enhanced binding in nonrelativistic QED, J. Math. Phys. 44 (2003), 1961-1970. Zbl 1062.81551 MR 1972757

[CFKS87] H. L. Cycon, R. G. Froese, W. Kirsch and B. Simon, Schrödinger operators with application to quantum mechanics and global geometry, Springer, 1987. Zbl 0619.47005 MR 0883643

[Dau83] I. Daubechies, An uncertainty principle for fermions with generalized kinetic energy, Comm. Math. Phys. 90 (1983), 511-520. Zbl 0946.81521 MR 0719431

[Dav77] E. B. Davies, Asymptotic analysis of some abstract evolution equations, J. Funct. Anal. 25 (1977), 81-101. Zbl 0347.47021 MR 0451031

[Dav79] _ Particle-boson interactions and the weak coupling limit, J. Math. Phys. 20 (1979), 345-351. MR 0525092

[Ger00] C. Gérard, On the existence of ground states for massless Pauli-Fierz Hamiltonians, Ann. Henri Poincaré 1 (2000), 443-459. Zbl 1004.81012 MR 1777307

[Ger06] A A remark on the paper: "On the existence of ground states for massless Pauli-Fierz Hamiltonians", mp_arc:06-146, preprint, 2006.

[GHPS12] C. Gérard, F. Hiroshima, A. Panati and A. Suzuki, Absence of ground state of the Nelson model with variable coefficients, J. Funct. Anal. 262 (2012), 273-299. Zbl 1238.81161 MR 2852262

[GLL01] M. Griesemer, E. Lieb and M. Loss, Ground states in non-relativistic quantum electrodynamics, Invent. Math. 145 (2001), 557-595. Zbl 1044.81133 MR 1856401

[HVV03] C. Hainzl, V. Vougalter and S. A. Vugalter, Enhanced binding in non-relativistic QED, Comm. Math. Phys. 233 (2003), 13-26. Zbl 1028.81056 MR 1957730

[Hir99] F. Hiroshima, Weak coupling limit removing an ultraviolet cut-off for a Hamiltonian of particles interacting with a quantized scalar field, J. Math. Phys. 40 (1999), 12151236. Zbl 0960.81017 MR 1674609

[Hir14] _ Functional integral approach to semi-relativistic Pauli-Fierz models, Adv. Math. 259 (2014), 784-840. Zbl 1290.81030 MR 3197672

[HS08] F. Hiroshima and I. Sasaki, Enhanced binding of an $N$-particle system interacting with a scalar field I, Math. Z. 259 (2008), 657-680. Zbl 1151.81016 MR 2395132

[HS01] F. Hiroshima and H. Spohn, Enhanced binding through coupling to a quantum field, Ann. Henri Poincaré 2 (2001), 1159-1187. Zbl 1020.81049 MR 1877237

[HSS11] F. Hiroshima, H. Spohn and A. Suzuki, The no-binding regime of the Pauli-Fierz model, J. Math. Phys. 52 (2011), 062104, 12 pp. Zbl 1317.81103 MR 2841742

[Nel64] E. Nelson, Interaction of nonrelativistic particles with a quantized scalar field, J. Math. Phys. 5 (1964), 1190-1197. MR 0175537

[Sas05] I. Sasaki, Ground state of the massless Nelson model in a non-Fock representation, J. Math. Phys. 46 (2005), 102107, 12 pp. Zbl 1111.81117 MR 2178579

[Spo98] H. Spohn, Ground state of a quantum particle coupled to a scalar Bose field, Lett. Math. Phys. 44 (1998), 9-16. Zbl 0908.60094 MR 1623746 